University of Nebraska - Lincoln

DigitalCommons@University of Nebraska - Lincoln

Faculty Publications, Department of Physics and Astronomy

Research Papers in Physics and Astronomy

$5-4-2005$

\title{
Nondipole effects in the triply differential cross section for double photoionization of $\mathrm{He}$
}

Andrei Y. Istomin

University of Nebraska-Lincoln, aistomin2@unl.edu

N. L. Manakov

Voronezh State University, manakov@phys.vsu.ru

A. V. Meremianin

Voronezh State University, meremianin@phys.vsu.ru

Anthony F. Starace

University of Nebraska-Lincoln, astarace1@unl.edu

Follow this and additional works at: https://digitalcommons.unl.edu/physicsfacpub

Part of the Physics Commons

Istomin, Andrei Y.; Manakov, N. L.; Meremianin, A. V.; and Starace, Anthony F., "Nondipole effects in the triply differential cross section for double photoionization of He" (2005). Faculty Publications, Department of Physics and Astronomy. 16.

https://digitalcommons.unl.edu/physicsfacpub/16

This Article is brought to you for free and open access by the Research Papers in Physics and Astronomy at DigitalCommons@University of Nebraska - Lincoln. It has been accepted for inclusion in Faculty Publications, Department of Physics and Astronomy by an authorized administrator of DigitalCommons@University of Nebraska Lincoln. 


\title{
Nondipole effects in the triply differential cross section for double photoionization of He
}

\author{
Andrei Y. Istomin, ${ }^{1}$ N. L. Manakov, ${ }^{2}$ A. V. Meremianin, ${ }^{2}$ and Anthony F. Starace ${ }^{1}$ \\ ${ }^{1}$ Department of Physics and Astronomy, The University of Nebraska, Lincoln, Nebraska 68588-0111, USA \\ ${ }^{2}$ Physics Department, Voronezh State University, Voronezh 394006, Russia
}

(Received 23 December 2004; published 4 May 2005)

\begin{abstract}
Lowest-order nondipole effects are studied systematically in double photoionization (DPI) of the He atom. $A b$ initio parametrizations of the quadrupole transition amplitude for DPI from the ${ }^{1} S_{0}$ state are presented in terms of the exact two-electron radial matrix elements. Analytic expressions for these matrix elements within lowest-order perturbation theory (LOPT) in the interelectron interaction are also given. The corresponding parametrizations for the dipole-quadrupole triply differential cross section (TDCS) are presented for the case of an elliptically polarized photon. A general analysis of retardation-induced asymmetries of the TDCS including the circular dichroism effect at equal energy sharing is presented. Numerical LOPT estimates of nondipole asymmetries in photoelectron angular distributions for the cases of linear and circular polarization and of the circular dichroism effect at equal energy sharing are presented. We find that experimental observation of nondipole effects at excess energies of the order of tens to hundreds of eV should be feasible in TDCS measurements. Our numerical results exhibit a nondipole forward-backward asymmetry in the TDCS for DPI of $\mathrm{He}$ at an excess energy of $450 \mathrm{eV}$ that is in qualitative agreement with existing experimental data.
\end{abstract}

DOI: 10.1103/PhysRevA.71.052702

PACS number(s): $32.80 . \mathrm{Fb}$

\section{INTRODUCTION}

Two major themes in vacuum ultraviolet (vuv) and soft$\mathrm{x}$-ray atomic photoionization studies in recent years are the analysis of nondipole (or retardation) effects in singleelectron photoionization (SPI) and the analysis of electron correlations in double photoionization (DPI), especially in the vuv range of photon energies. The SPI angular distributions in the electric-dipole approximation (EDA) involve only a single polarization and angle-dependent parameter, $|\mathbf{e} \cdot \mathbf{p}|^{2}$, composed of the photon polarization vector $\mathbf{e}\left(\mathbf{e} \cdot \mathbf{e}^{*}\right.$ $=1$ ) and the photoelectron momentum p. Therefore, retardation effects, which imply a dependence of the SPI cross section on the photon wave vector, $\mathbf{k}$, by means of the scalar product $(\mathbf{k} \cdot \mathbf{p})$, do not affect the polarization dependence of photoelectron angular distributions, and result only in a spatial asymmetry of the photoelectron angular distributions with respect to the direction of $\mathbf{k}$ (the forward-backward asymmetry), which originates from the interference between dipole and nondipole SPI amplitudes. Although the first nondipole SPI measurements for Ar were in the x-ray region [1], recently the forward-backward asymmetry has been found to be significant also in the vuv region (see, e.g., results for Xe at $E_{\gamma} \lesssim 200 \mathrm{eV}$ [2-4] and for $\mathrm{He}$ at $E_{\gamma} \lesssim 160 \mathrm{eV}$ [5]). As shown in Ref. [6], not only lowest-order (quadrupole) but also higher-order (octupole) nondipole effects should be taken into account in order to explain experimental results on neon valence photoemission at relatively low photon energies $E_{\gamma} \lesssim 1 \mathrm{keV}$. Besides the spatial asymmetry in the angular distributions, the theory predicts also new retardationinduced features in SPI for spin-resolved photoelectron measurements [7] (which have been recently observed experimentally [8]) and for the case of polarized atoms [9].

The process of DPI has attracted much theoretical and experimental interest in view of its importance for analyzing electron correlations. (For recent reviews, see Refs. [10,11].)
Most experiments concern the He atom, in which DPI represents the prototype for three-body fragmentation of a Coulomb system by a single photon. The photon energies employed typically range between 100 and $530 \mathrm{eV}$. Measurements of the triply differential cross sections (TDCSs) at these energies have been guided by theoretical predictions using the EDA. Recent advances in experimental techniques allow the measurement of even very small TDCSs of the order of $\left(10^{-2}-10^{-3}\right) \mathrm{b} \mathrm{eV}^{-1} \mathrm{sr}^{-2}$ [12]. Despite the fact that DPI is essentially a many-body, correlationinduced process, a number of theoretical approaches (mostly using numerically intensive techniques to account for correlations in the two-electron continuum) allow one to obtain excellent agreement with absolute experimental data for the TDCSs. The accuracy of such calculations is thus comparable to that for SPI. A number of questions have been addressed in studies of DPI within the EDA framework, such as the photoelectron angular distributions for linearly and circularly polarized light for various excess energies and energy sharings (see, e.g., Refs. [10,11]), along with attempts to identify the dominant physical mechanisms in particular energy regimes [12-16].

In contrast to SPI, existing information on nondipole effects in DPI is very sparse. To the best of our knowledge, there are only a few works that have considered nondipole effects in DPI of He [17] and highly charged He-like ions $[18,19]$. However, these studies are for photon energies in excess of $1 \mathrm{keV}$, and at such high photon energies (and therefore high excess energies) the DPI process has not been investigated by coincidence measurements. Also, these works treat nondipole effects only in the doubly differential cross sections, which exhibit angular dependencies that are similar to those for SPI, and thus the question of the importance of nondipole effects on the TDCS, the most informative observable of the DPI process, is open. The lack of theoretical analyses of nondipole effects in the TDCSs may be explained 
by two circumstances. First, even for the case of dipole DPI, an accurate account of electron correlations requires timeconsuming numerical approaches that have only been developed relatively recently. Second, existing experimental data do not exhibit significant deviations from theoretical predictions based on the EDA. However, nearly all published TDCS measurements were performed in the perpendicular plane geometry, in which the photoelectron detection plane is orthogonal to the direction of the photon beam. In lowest order, retardation corrections stem from terms $\sim(\mathbf{k} \cdot \mathbf{r})$ in the power series expansion of the vector potential. Therefore, as for SPI, they enter the DPI amplitude and the TDCS only through scalar products $\left(\mathbf{k} \cdot \mathbf{p}_{1}\right)$ and $\left(\mathbf{k} \cdot \mathbf{p}_{2}\right)$, which obviously vanish for the perpendicular plane geometry.

In general, it may be expected that an account of retardation corrections to the dipole amplitude of DPI should result in more diverse asymmetries of the TDCS than those for angular distributions in SPI. Indeed, for the case of DPI, along with the asymmetry of the TDCS with respect to inversion of the direction of the photon wave vector $\mathbf{k}$ (as in SPI), nondipole corrections also modify the asymmetry of the TDCS with respect to inversion of the photon helicity, i.e., the circular dichroism (CD) effect. This effect, predicted first theoretically in Ref. [20] (and then observed experimentally [21]), has attracted much attention, both theoretical and experimental [10,22]. Specifically, the photon-helicitydependent CD term in the TDCS originates from an interference between real and imaginary parts of particular components of the (generally non-Hermitian) DPI amplitude; the $\mathrm{CD}$ effect thus permits direct experimental measurement of this otherwise elusive "cross-interference." Up to now, all theoretical treatments of $\mathrm{CD}$ have employed the EDA $[10,20,22-29]$ and their predictions are generally in agreement with existing experiments [28-32]. Although these measurements do not show any signatures of nondipole effects, this fact is expected because all of them have been performed in the perpendicular plane geometry, in which the lowest-order retardation effects vanish. As a phenomenological analysis (based only on rotational-invariance and symmetry arguments) shows [24], the existence of CD in DPI is possible since in this case (in contrast to SPI) we have two momentum vectors, $\mathbf{p}_{1}$ and $\mathbf{p}_{2}$, and thus the dichroic factor, $\xi\left(\hat{\mathbf{k}} \cdot\left[\hat{\mathbf{p}}_{1} \times \hat{\mathbf{p}}_{2}\right]\right)$ (where $\xi$ is the degree of circular polarization of the photon beam), enters the TDCS multiplied by a scalar factor $\alpha_{\mathrm{CD}}\left(p_{1}, p_{2}, \cos \theta\right)$, where $\cos \theta \equiv\left(\hat{\mathbf{p}}_{1} \cdot \hat{\mathbf{p}}_{2}\right)$. A distinct feature of the CD effect within the EDA is that it vanishes at equal energy sharing $\left(\alpha_{\mathrm{CD}}=0\right.$ at $\left.p_{1}=p_{2}\right)$ : for DPI from a ${ }^{1} S_{0}$ state, the EDA amplitude is described by a single scalar function, $f\left(p_{1}, p_{2}, \cos \theta\right)$, so that $\alpha_{\mathrm{CD}}=\operatorname{Im}\left\{f\left(p_{1}, p_{2}\right.\right.$, $\left.\cos \theta) f^{*}\left(p_{2}, p_{1}, \cos \theta\right)\right\}$. [This form of $\alpha_{\mathrm{CD}}$ follows from the invariance of the TDCS (and thus of the total CD term) with respect to interchange of photoelectrons, i.e., the substitution $\mathbf{p}_{1} \rightleftarrows \mathbf{p}_{2}$.] This rule fails if one treats the electron-photon interaction beyond the EDA, since, if one introduces the dependence of the TDCS upon the wave vector of the incident light (or, equivalently, upon its spatial inhomogeneity), the symmetry of the problem is reduced. Therefore, the DPI amplitude involves [along with $f\left(p_{1}, p_{2}, \cos \theta\right)$ and $f\left(p_{2}\right.$, $\left.\left.p_{1}, \cos \theta\right)\right]$ additional dynamical parameters which account for retardation effects, and the $\mathrm{CD}$ factor $\alpha_{\mathrm{CD}}$ acquires a nondipole correction, which does not vanish at $p_{1}=p_{2}$.

Recently, we have reported the first predictions of nondipole effects in the TDCS for DPI, for both linearly and circularly polarized light $[33,34]$. Here we present a more detailed account of our analyses. First, we derive a general, model-independent parametrization for the amplitude of DPI from a singlet ${ }^{1} S_{0}$ state, taking into account lowest-order retardation corrections to the electron-photon interaction operator in terms of the two-electron reduced matrix elements. Second, we derive the corresponding parametrizations for the dipole-quadrupole (E1-E2) TDCS for the cases of linearly and circularly polarized light, as well as for the general case of elliptic polarization. Then, we discuss the nondipole effects (i.e., originating from interference of the E1 and E2 amplitudes) to be expected in the TDCS, such as (i) asymmetry with respect to inversion of the direction of the wave vector $\mathbf{k}$; (ii) asymmetry with respect to reflection of the photoelectron pair in the polarization plane, and (iii) asymmetry with respect to inversion of the photon helicity, i.e., the $\mathrm{CD}$ effect. In particular, we discuss the $\mathrm{CD}$ effect at equal energy sharing. Using lowest-order perturbation theory (LOPT) to account for interelectron correlations [16], we estimate the magnitudes of these effects for DPI of He at excess energies ranging from tens to hundreds of $\mathrm{eV}$. In an Appendix, we present our analytic results for the LOPT limit of the exact two-electron reduced matrix elements of the quadrupole operator that enter our $a b$ initio parametrization of the DPI amplitude.

\section{AB INITIO PARAMETRIZATIONS OF THE DPI TRANSITION AMPLITUDE AND TDCS}

\section{A. Velocity and length gauge expressions for the lowest-order retardation corrections}

In order to parametrize the DPI amplitude in a unified way that is valid for both the velocity and length forms of the electron-photon interaction, we shall first present explicit analytic expressions for the leading retardation corrections to the electric-dipole electron-photon interaction in the velocity and length gauges. Our approach is nonrelativistic and starts from the standard form for the electron-photon interaction in the Coulomb gauge for the four-potential $A^{\mu}=(\Phi=0, \mathbf{A})$ [35],

$$
V(\mathbf{r}, t)=-\frac{e}{m c}(\mathbf{A}(\mathbf{r}, t) \cdot \mathbf{p}),
$$

where $\mathbf{p}=-i \hbar \boldsymbol{\nabla}_{\mathbf{r}}$ is the momentum operator, and $e=-|e|, m$, and $c$ are the electron charge, the electron mass, and the speed of light. Since we are interested in a one-photon photoionization process, the vector potential has the form $\mathbf{A}(\mathbf{r}, t)$ $=\mathbf{e} e^{i(\mathbf{k} \cdot \mathbf{r}-\omega t)}$, where $\omega$ is the photon frequency, $\mathbf{k}=\omega \hat{\mathbf{k}} / c$ is the wave vector, and $\mathbf{e}\left[\left(\mathbf{e} \cdot \mathbf{e}^{*}\right)=1\right]$ is the polarization vector. For simplicity, we take the amplitude of the potential to be equal to unity.

The long-wave limit of matrix elements involving $V(\mathbf{r}, t)$ implies an expansion of the four-potential $A^{\mu}$ in powers of $(\mathbf{k} \cdot \mathbf{r})$. Below, we restrict our consideration to only the 
lowest-order retardation corrections to the EDA, i.e., to terms of order $k$, so that

$$
\begin{gathered}
V(\mathbf{r}, t) \approx V^{(V)} \exp (-i \omega t), \\
V^{(V)}=-\frac{e}{m c}\left[(\mathbf{e} \cdot \mathbf{p})+i \frac{\omega}{c}(\mathbf{e} \cdot \mathbf{p})(\hat{\mathbf{k}} \cdot \mathbf{r})\right] .
\end{gathered}
$$

The first term in $V^{(V)}$ is the standard velocity form for the electric-dipole interaction while the second term corresponds to the velocity form for retardation corrections. In order to analyze matrix elements using angular momentum techniques (such as the Wigner-Eckart theorem, etc.) [36], it is convenient to represent the expression $(\mathbf{e} \cdot \mathbf{p})(\hat{\mathbf{k}} \cdot \mathbf{r})$ as a scalar product of two irreducible tensors involving either photon (e and $\hat{\mathbf{k}}$ ) or electron (p and $\mathbf{r}$ ) parameters. This transformation follows from standard formulas for irreducible tensor products of several vectors [36] and allows the operator $V^{(V)}$ to be written as

$$
V^{(V)}=V_{d}+V_{q}+V_{m},
$$

where

$$
\begin{gathered}
V_{d}=-\frac{e}{m c}(\mathbf{e} \cdot \mathbf{p}), \\
V_{q}=-i \frac{e \omega}{m c^{2}}\left(\{\hat{\mathbf{k}} \otimes \mathbf{e}\}_{2} \cdot\{\mathbf{r} \otimes \mathbf{p}\}_{2}\right), \\
V_{m}=-i \frac{e \omega}{2 m c^{2}}([\hat{\mathbf{k}} \times \mathbf{e}] \cdot[\mathbf{r} \times \mathbf{p}]) .
\end{gathered}
$$

The term $V_{m}$ involves the orbital angular momentum operator, $\mathbf{l}=[\mathbf{r} \times \mathbf{p}]$, and thus describes the orbital magnetic-dipole interaction.

To obtain the length form counterpart of the operator $V^{(V)}$ in Eq. (4), we perform the following gauge transformation of the four-potential $A^{\mu}=(0, \mathbf{e}(1+i \mathbf{k} \cdot \mathbf{r}) \exp (-i \omega t))$ :

$$
\mathbf{A}^{\prime}=\mathbf{A}+\nabla f(\mathbf{r}, t), \quad \Phi^{\prime}=\Phi-\frac{1}{c} \frac{\partial f(\mathbf{r}, t)}{\partial t},
$$

where $f(\mathbf{r}, t)$ is given by

$$
f(\mathbf{r}, t)=-(\mathbf{e} \cdot \mathbf{r})(1+i \mathbf{k} \cdot \mathbf{r} / 2) e^{-i \omega t} .
$$

We thus obtain

$$
\Phi^{\prime}(\mathbf{r}, t)=-i \frac{\omega}{c}(\mathbf{e} \cdot \mathbf{r})[1+i(\mathbf{k} \cdot \mathbf{r}) / 2] e^{-i \omega t},
$$

$$
\mathbf{A}^{\prime}(\mathbf{r}, t)=\frac{i}{2}[\mathbf{e}(\mathbf{k} \cdot \mathbf{r})-\mathbf{k}(\mathbf{e} \cdot \mathbf{r})] e^{-i \omega t}=\frac{i}{2}[[\mathbf{k} \times \mathbf{e}] \times \mathbf{r}] e^{-i \omega t}
$$

The length form counterpart to Eq. (3) for the electronphoton interaction operator to leading order in the retardation corrections is therefore

$$
V^{\prime}(\mathbf{r}, t)=-\frac{e}{m c}\left(\mathbf{A}^{\prime}(\mathbf{r}, t) \cdot \mathbf{p}\right)+e \Phi^{\prime}(\mathbf{r}, t)=V^{(L)} e^{-i \omega t},
$$

where

$$
V^{(L)}=-i \frac{e \omega}{c}\left[(\mathbf{e} \cdot \mathbf{r})+i \frac{\omega}{2 c}(\mathbf{e} \cdot \mathbf{r})(\hat{\mathbf{k}} \cdot \mathbf{r})+\frac{1}{2 m c}([\hat{\mathbf{k}} \times \mathbf{e}] \cdot \mathbf{l})\right] .
$$

Similarly to Eqs. (4) and (5), we rewrite the expression above in tensor form,

$$
\begin{gathered}
V^{(L)}=V_{d}^{\prime}+V_{q}^{\prime}+V_{m}^{\prime}, \\
V_{d}^{\prime}=-i \frac{e \omega}{c}(\mathbf{e} \cdot \mathbf{r}), \\
V_{q}^{\prime}=\frac{e \omega^{2}}{2 c^{2}}(\mathbf{e} \cdot \mathbf{r})(\hat{\mathbf{k}} \cdot \mathbf{r})=\frac{e \omega^{2}}{2 c^{2}} r^{2}\left(\{\hat{\mathbf{k}} \otimes \mathbf{e}\}_{2} \cdot\{\hat{\mathbf{r}} \otimes \hat{\mathbf{r}}\}_{2}\right), \\
V_{m}^{\prime}=-i \frac{e \omega}{2 m c^{2}}([\hat{\mathbf{k}} \times \mathbf{e}] \cdot \mathbf{l}) .
\end{gathered}
$$

Note that $\{\hat{\mathbf{r}} \otimes \hat{\mathbf{r}}\}_{2 m}=\sqrt{2 / 3} C_{2 m}(\hat{\mathbf{r}}), \quad$ where $\quad C_{l m}(\hat{\mathbf{r}})$ $\equiv \sqrt{4 \pi /(2 l+1)} Y_{l m}(\hat{\mathbf{r}})$ is the modified spherical harmonic [36].

Because our treatment accounts for the leading corrections to the EDA, the lowest-order spin effects should also be accounted for. This can be done either by substituting $\mathbf{p} \rightarrow(\mathbf{p}+i[\mathbf{s} \times \mathbf{k}])$ (where $\mathbf{s}$ is the electron spin operator) in Eq. (1) [35], or, in lowest order, by simply making the substitution $\mathbf{l} \rightarrow(\mathbf{l}+2 \mathbf{s})$ in the magnetic-dipole terms $\left(V_{m}=V_{m}^{\prime}\right)$ in Eqs. (5) and (12).

\section{B. Parametrizations of the DPI amplitude with account of dipole and quadrupole terms}

We consider DPI from the singlet ${ }^{1} S_{0}$ state $|0\rangle$ in the nonrelativistic domain of photon energies taking into account lowest-order retardation corrections. For DPI from a ${ }^{1} S_{0}$ state, neither orbital nor spin-dependent parts of the magnetic dipole interaction contribute to the transition amplitude (see Ref. [19] for details). Therefore, the magnetic-dipole operator in Eqs. (5) and (12) as well as the spin dependence of the two-electron wave functions are suppressed in our analysis of the transition amplitude $A$ to the final two-electron singlet state, $\left|\mathbf{p}_{1} \mathbf{p}_{2}\right\rangle$, with asymptotic electron momenta $\mathbf{p}_{1}$ and $\mathbf{p}_{2}$. The dipole-quadrupole TDCS for DPI is

$$
\frac{d^{3} \sigma}{d \epsilon_{1} d \Omega_{1} d \Omega_{2}} \equiv \sigma=\mathcal{A}|A|^{2},
$$

where $\mathcal{A}=4 \pi^{2} \alpha p_{1} p_{2} / \omega$ is a normalization factor, and $\alpha$ $=1 / 137$. Atomic units are used throughout the rest of this paper. The amplitude $A$ involving E1 and E2 components has the unified form

$$
A=A_{d}+A_{q}=\left\langle\mathbf{p}_{1} \mathbf{p}_{2}\left|(\mathbf{e} \cdot \mathbf{D})+\left(\{\hat{\mathbf{k}} \otimes \mathbf{e}\}_{2} \cdot Q_{2}\right)\right| 0\right\rangle
$$

for both velocity and length gauges of the electron-photon interaction. In the velocity gauge, $\mathbf{D} \equiv \mathbf{D}^{(V)}=-i\left(\boldsymbol{\nabla}_{1}+\boldsymbol{\nabla}_{2}\right)$ and 
$Q_{2 m} \equiv Q_{2 m}^{(V)}=\alpha \omega\left(\left\{\mathbf{r}_{1} \otimes \nabla_{1}\right\}_{2 m}+\left\{\mathbf{r}_{2} \otimes \nabla_{2}\right\}_{2 m}\right) . \quad$ In the length gauge, $\mathbf{D} \equiv \mathbf{D}^{(L)}=i \omega\left(\mathbf{r}_{1}+\mathbf{r}_{2}\right)$ and $Q_{2 m} \equiv Q_{2 m}^{(L)}=-(1 / 2) \alpha \omega^{2}\left(\left\{\mathbf{r}_{1}\right.\right.$ $\left.\left.\otimes \mathbf{r}_{1}\right\}_{2 m}+\left\{\mathbf{r}_{2} \otimes \mathbf{r}_{2}\right\}_{2 m}\right)$. Because the amplitude $A$ in Eq. (14) is a scalar (i.e., it is independent of magnetic quantum numbers), a rotationally invariant parametrization may be given in terms of the vectors of the problem and scalar dynamical parameters dependent on $p_{1}, p_{2}$, and the mutual angle $\theta_{12}$ $\equiv \theta$ between $\mathbf{p}_{1}$ and $\mathbf{p}_{2}$, i.e., $\cos \theta=\left(\hat{\mathbf{p}}_{1} \cdot \hat{\mathbf{p}}_{2}\right)$.

The parametrization of the EDA amplitude $A_{d}$ in terms of scalar products of the vectors $\mathbf{e}, \hat{\mathbf{p}}_{1}$, and $\hat{\mathbf{p}}_{2}$ is well known $[10,24]$,

$$
A_{d}=f_{1}\left(\mathbf{e} \cdot \hat{\mathbf{p}}_{1}\right)+f_{2}\left(\mathbf{e} \cdot \hat{\mathbf{p}}_{2}\right) .
$$

In this equation, $f_{1} \equiv f\left(p_{1}, p_{2}, \cos \theta\right)$ and $f_{2} \equiv f\left(p_{2}, p_{1}, \cos \theta\right)$ are defined by a single function,

$$
\begin{aligned}
f\left(p, p^{\prime}, \cos \theta\right)= & \sum_{l=1}^{\infty}(-1)^{l+1}\left[\sum_{l^{\prime}=l \pm 1} D_{l l^{\prime}}\left(p, p^{\prime}\right)\right] \\
& \times P_{l}^{(1)}(\cos \theta),
\end{aligned}
$$

where $P_{l}^{(n)}(x)$ is the $n$th derivative of the Legendre polynomial $P_{l}(x), P_{l}^{(n)}(x)=\left(d^{n} / d x^{n}\right) P_{l}(x)$. The energy-dependent coefficient $D_{l l^{\prime}}\left(p, p^{\prime}\right)$ is given by

$$
D_{l l^{\prime}}\left(p, p^{\prime}\right)=\frac{\left\langle p p^{\prime} ;\left(l l^{\prime}\right) 1\|\mathbf{D}\| 0\right\rangle}{\sqrt{(2 l+1)\left(2 l^{\prime}+1\right) \max \left(l, l^{\prime}\right)}},
$$

where $\left\langle p p^{\prime} ;\left(l l^{\prime}\right) 1\|\mathbf{D}\| 0\right\rangle$ is the reduced matrix element of the operator $\mathbf{D}$ between the ${ }^{1} S_{0}$ state and the $P$-wave component of the two-electron continuum state $\left|\mathbf{p p}^{\prime}\right\rangle$, with photoelectron angular momenta $l$ and $l^{\prime}=l \pm 1$.

In order to derive a model-independent parametrization of the quadrupole amplitude $A_{q}$ (i.e., to establish its dependence on the photon parameters $\mathbf{e}$ and $\mathbf{k}$ and the angle $\theta$ between $\mathbf{p}_{1}$ and $\mathbf{p}_{2}$ ), we employ techniques similar to those that have been developed for parametrization of the electric dipole TDCS [24]. First, we use the well-known multipole expansion of the final state $\left|\mathbf{p}_{1} \mathbf{p}_{2}\right\rangle$ in terms of bipolar harmonics $\mathcal{C}_{L M}^{l l^{\prime}}\left(\hat{\mathbf{p}}, \hat{\mathbf{p}}^{\prime}\right)$,

$$
\left|\mathbf{p}_{1} \mathbf{p}_{2}\right\rangle=\sum_{l_{1} l_{2} l m} \mathcal{C}_{l m}^{l_{1} l_{2} *}\left(\hat{\mathbf{p}}_{1}, \hat{\mathbf{p}}_{2}\right)\left|p_{1} p_{2} ;\left(l_{1} l_{2}\right) l m\right\rangle,
$$

where

$$
\begin{aligned}
\mathcal{C}_{l m}^{l_{1} l_{2}}\left(\hat{\mathbf{p}}_{1}, \hat{\mathbf{p}}_{2}\right) & =\sum_{m_{1}, m_{2}} \mathcal{C}_{l_{1} m_{1} l_{2} m_{2}}^{l m} \mathcal{C}_{l_{1} m_{1}}\left(\hat{\mathbf{p}}_{1}\right) \mathcal{C}_{l_{2} m_{2}}\left(\hat{\mathbf{p}}_{2}\right) \\
& =\left\{\mathcal{C}_{l_{1} m_{1}}\left(\hat{\mathbf{p}}_{1}\right) \otimes \mathcal{C}_{l_{2} m_{2}}\left(\hat{\mathbf{p}}_{2}\right)\right\}_{l m},
\end{aligned}
$$

and $\mathcal{C}_{l_{1} m_{1} l_{2} m_{2}}^{l m}$ denotes a Clebsch-Gordon coefficient. Using the Wigner-Eckart theorem [36], the polarization-angular dependence of $A_{q}$ may be given in terms of a sum of scalar products of two rank-2 tensors,

$$
\begin{aligned}
A_{q}= & \frac{1}{\sqrt{5}} \sum_{l_{1} l_{2}}\left(\{\hat{\mathbf{k}} \otimes \mathbf{e}\}_{2} \cdot \mathcal{C}_{2}^{l_{1} l_{2}}\left(\hat{\mathbf{p}}_{1}, \hat{\mathbf{p}}_{2}\right)\right) \\
& \times\left\langle p_{1} p_{2} ;\left(l_{1} l_{2}\right) 2\left\|Q_{2}\right\| 0\right\rangle,
\end{aligned}
$$

where $\left\langle p_{1} p_{2} ;\left(l_{1} l_{2}\right) 2\left\|Q_{2}\right\| 0\right\rangle$ is the reduced matrix element of the operator $Q_{2 m}$ between the initial ${ }^{1} S_{0}$ state, $|0\rangle$, and the $D$-wave component of $\left|\mathbf{p}_{1} \mathbf{p}_{2}\right\rangle$ with photoelectron angular momenta $l_{1}$ and $l_{2}=l_{1}, l_{1} \pm 2$.

Taking symmetry requirements into account, Eq. (20) may be simplified. Since the bipolar harmonics in this equation are rank-2 irreducible tensors composed of the vectors $\hat{\mathbf{p}}_{1}$ and $\hat{\mathbf{p}}_{2}$, they may be represented as a superposition of all possible rank-2 irreducible tensors composed of $\hat{\mathbf{p}}_{1}$ and $\hat{\mathbf{p}}_{2}$. There are three such independent tensors: $\left\{\hat{\mathbf{p}}_{1} \otimes \hat{\mathbf{p}}_{1}\right\}_{2 m},\left\{\hat{\mathbf{p}}_{2}\right.$ $\left.\otimes \hat{\mathbf{p}}_{2}\right\}_{2 m}$, and $\left\{\hat{\mathbf{p}}_{1} \otimes \hat{\mathbf{p}}_{2}\right\}_{2 m}$. Thus phenomenologically the amplitude $A_{q}$ may be written as

$$
\begin{aligned}
A_{q}= & \{\mathbf{e} \otimes \hat{\mathbf{k}}\}_{2} \cdot\left(g_{1}\left\{\hat{\mathbf{p}}_{1} \otimes \hat{\mathbf{p}}_{1}\right\}_{2}+g_{2}\left\{\hat{\mathbf{p}}_{2} \otimes \hat{\mathbf{p}}_{2}\right\}_{2}\right. \\
& \left.+2 g_{s}\left\{\hat{\mathbf{p}}_{1} \otimes \hat{\mathbf{p}}_{2}\right\}_{2}\right),
\end{aligned}
$$

where we have introduced the generally complex parameters $g_{1,2}$ and $g_{s}$, which depend on $p_{1}, p_{2}$, and $\theta$; the factor 2 is introduced for the sake of convenience. After expressing the scalar products of rank-2 tensors in this identity in terms of the Cartesian scalar products of vectors of the problem (see, e.g., Sec. 3.2 of Ref. [36]) and taking into account the invariance of the amplitude with respect to interchange of the photoelectrons (i.e., $\mathbf{p}_{1} \rightleftarrows \mathbf{p}_{2}$ ), we arrive at our final parametrization of the DPI quadrupole transition amplitude,

$$
\begin{aligned}
A_{q}= & g_{1}\left(\mathbf{e} \cdot \hat{\mathbf{p}}_{1}\right)\left(\hat{\mathbf{p}}_{1} \cdot \hat{\mathbf{k}}\right)+g_{2}\left(\mathbf{e} \cdot \hat{\mathbf{p}}_{2}\right)\left(\hat{\mathbf{p}}_{2} \cdot \hat{\mathbf{k}}\right) \\
& +g_{s}\left[\left(\mathbf{e} \cdot \hat{\mathbf{p}}_{1}\right)\left(\hat{\mathbf{p}}_{2} \cdot \hat{\mathbf{k}}\right)+\left(\mathbf{e} \cdot \hat{\mathbf{p}}_{2}\right)\left(\hat{\mathbf{p}}_{1} \cdot \hat{\mathbf{k}}\right)\right],
\end{aligned}
$$

in terms of only two functions: $g_{s} \equiv g_{s}\left(p_{1}, p_{2}, \cos \theta\right)$ $=g_{s}\left(p_{2}, p_{1}, \cos \theta\right)$ (which is symmetric in the arguments $p_{1}$ and $\left.p_{2}\right)$ and the function $g\left(p, p^{\prime}, \cos \theta\right)$, with $g_{1}$ $\equiv g\left(p_{1}, p_{2}, \cos \theta\right)$ and $g_{2} \equiv g\left(p_{2}, p_{1}, \cos \theta\right)$.

The explicit forms of the functions $g\left(p, p^{\prime}, \cos \theta\right)$ and $g_{s}\left(p, p^{\prime}, \cos \theta\right)$ in terms of the reduced matrix elements introduced in Eq. (20) may be established by using the reduction formulas for the rank-2 bipolar harmonics in Eq. (20) that are derived in Ref. [24]. The final expressions for $g$ and $g_{s}$ are [33]

$$
\begin{aligned}
g_{s}\left(p, p^{\prime}, \cos \theta\right)= & \sum_{l=1}^{\infty}(-1)^{l+1}\left[\sum_{l^{\prime}=l \pm 2} Q_{l l^{\prime}}\left(p, p^{\prime}\right) P_{\left(l+l^{\prime}\right) / 2}^{(2)}(\cos \theta)\right. \\
& +\sqrt{6} Q_{l l}\left(p, p^{\prime}\right) \\
& \left.\times\left(P_{l+1}^{(2)}(\cos \theta)-\frac{2 l+3}{2} P_{l}^{(1)}(\cos \theta)\right)\right],
\end{aligned}
$$

$$
\begin{aligned}
g\left(p, p^{\prime}, \cos \theta\right)= & \sum_{l=2}^{\infty}(-1)^{l}\left[\sum_{l^{\prime}=l \pm 2} Q_{l l^{\prime}}\left(p, p^{\prime}\right)+\sqrt{6} Q_{l l}\left(p, p^{\prime}\right)\right] \\
& \times P_{l}^{(2)}(\cos \theta),
\end{aligned}
$$

where

$$
Q_{l l^{\prime}}\left(p, p^{\prime}\right)=\sqrt{\frac{4\left(l+l^{\prime}-2\right) !}{\left(l+l^{\prime}+3\right) !}}\left\langle p p^{\prime} ;\left(l l^{\prime}\right) 2\left\|Q_{2}\right\| 0\right\rangle .
$$


1. Symmetrized quadrupole amplitudes: $g_{+}^{(\mathrm{g})}, g_{-}^{(\mathrm{g})}$, and $g^{(\mathrm{u})}$

Another parametrization of the DPI quadrupole amplitude $A_{q}$, which is similar to the one commonly used in the literature within the EDA framework, is the parametrization in terms of symmetrized combinations of $\hat{\mathbf{p}}_{1}$ and $\hat{\mathbf{p}}_{2}: \mathbf{p}_{+}=\left(\hat{\mathbf{p}}_{1}\right.$ $\left.+\hat{\mathbf{p}}_{2}\right) / 2$ and $\mathbf{p}_{-}=\left(\hat{\mathbf{p}}_{1}-\hat{\mathbf{p}}_{2}\right) / 2$ [which are orthogonal, $\mathbf{p}_{+} \cdot \mathbf{p}_{-}$ $=\mathbf{0}$, and have the following moduli: $p_{+}=\cos (\theta / 2)$ and $p_{-}$ $=\sin (\theta / 2)]$. With these definitions, Eqs. (15) and (21) have the following form:

$$
\begin{gathered}
A_{d}=f^{(\mathrm{g})}\left(\mathbf{e} \cdot \mathbf{p}_{+}\right)+f^{(\mathrm{u})}\left(\mathbf{e} \cdot \mathbf{p}_{-}\right), \\
A_{q}=g_{+}^{(\mathrm{g})}\left(\mathbf{e} \cdot \mathbf{p}_{+}\right)\left(\hat{\mathbf{k}} \cdot \mathbf{p}_{+}\right)+g_{-}^{(\mathrm{g})}\left(\mathbf{e} \cdot \mathbf{p}_{-}\right)\left(\hat{\mathbf{k}} \cdot \mathbf{p}_{-}\right) \\
+g^{(\mathrm{u})}\left[\left(\mathbf{e} \cdot \mathbf{p}_{+}\right)\left(\hat{\mathbf{k}} \cdot \mathbf{p}_{-}\right)+\left(\mathbf{e} \cdot \mathbf{p}_{-}\right)\left(\hat{\mathbf{k}} \cdot \mathbf{p}_{+}\right)\right],
\end{gathered}
$$

where the symmetrized amplitudes are $f^{(\mathrm{g})}=f_{1}+f_{2}, f^{(\mathrm{u})}=f_{1}$ $-f_{2}, g_{ \pm}^{(\mathrm{g})}=g_{1}+g_{2} \pm 2 g_{s}$, and $g^{(\mathrm{u})}=g_{1}-g_{2}$. For equal energy sharing, $f^{(\mathrm{u})}=g^{(\mathrm{u})}=0$, and the parametrization in terms of the symmetrized amplitudes becomes particularly convenient. The exact expressions for our symmetrized amplitudes in terms of Legendre polynomials and reduced matrix elements follow immediately from those for the functions $f\left(p, p^{\prime}, \cos \theta\right)$ and $g\left(p, p^{\prime}, \cos \theta\right), g_{s}\left(p, p^{\prime}, \cos \theta\right)$ defined in Eqs. (16) and (22). The amplitudes $f^{(\mathrm{u})}$ and $g^{(\mathrm{u})}$ have simple forms,

$$
\begin{aligned}
f^{(\mathrm{u})} & =\sum_{l=1}^{\infty}(-1)^{l+1} \sum_{l^{\prime}=l \pm 1}\left[D_{l l^{\prime}}\left(p, p^{\prime}\right)-D_{l l^{\prime}}\left(p^{\prime}, p\right)\right] P_{l}^{(1)}(\cos \theta), \\
g^{(\mathrm{u})} & =\sum_{l=2}^{\infty}(-1)^{l} \sum_{l^{\prime}=l \pm 2}\left[Q_{l l^{\prime}}\left(p, p^{\prime}\right)-Q_{l l^{\prime}}\left(p^{\prime}, p\right)\right] P_{l}^{(2)}(\cos \theta) .
\end{aligned}
$$

[We note that the description of the dipole amplitude $A_{d}$ given above is very similar to its well-known parametrization in terms of the vectors $\hat{\mathbf{p}}_{1} \pm \hat{\mathbf{p}}_{2}$ and the symmetrized "gerade" and "ungerade" amplitudes $a_{\mathrm{g}, \mathrm{u}}=\left(f_{1} \pm f_{2}\right) / 2$ [10,25,37]; note that $f^{(\mathrm{g}, \mathrm{u})}=2 a_{\mathrm{g}, \mathrm{u}}$.]

The results in Eqs. (15)-(17) and (21)-(26) are general and give $a b$ initio parametrizations of the dipole and quadrupole DPI amplitudes, independent of the dynamical model used for calculations of the reduced matrix elements (17) and (23). Thus, the measurements of nondipole effects in DPI of He allow one to probe electron correlations in the $D$-wave part of the two-electron continuum state $\left|\mathbf{p}_{1} \mathbf{p}_{2}\right\rangle$ (while only the $P$-wave part contributes to the EDA amplitude $A_{d}$ ).

\section{Model-independent parametrizations for the dipole-quadrupole TDCS}

We present here parametrizations for the TDCS defined by Eqs. (13)-(15) and (21) for the cases of linear, circular, and elliptic polarization, and discuss the nondipole effects to be expected in measurements.

\section{Linear polarization}

For the case of DPI by linearly polarized light described by the real photon polarization vector $\mathbf{e} \equiv \hat{\boldsymbol{\epsilon}}$, the dipole-

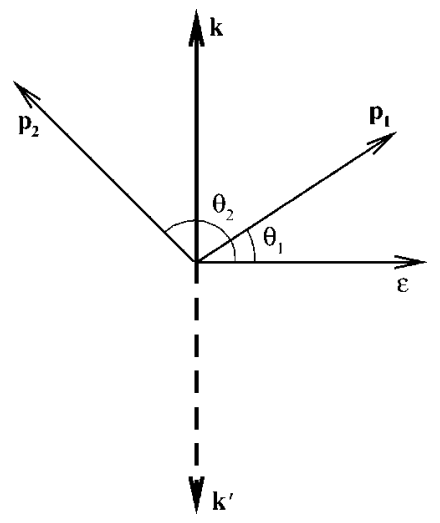

FIG. 1. Geometry suitable for observation of retardation effects in the TDCS for DPI by linearly polarized light. The vectors $\mathbf{p}_{1}, \mathbf{p}_{2}$, $\boldsymbol{\epsilon}$, and $\mathbf{k}$ lie in one plane.

quadrupole TDCS may be presented in terms of three real parameters (upon neglecting the small E2-E2 terms),

$$
\sigma=\sigma_{d}+\mathcal{A}\left[\alpha_{1}\left(\hat{\mathbf{p}}_{1} \cdot \hat{\mathbf{k}}\right)+\alpha_{2}\left(\hat{\mathbf{p}}_{2} \cdot \hat{\mathbf{k}}\right)\right],
$$

where $\sigma_{d}=\mathcal{A}\left|A_{d}\right|^{2}$ is the dipole TDCS, and the two real, $\hat{\mathbf{k}}$-independent parameters $\alpha_{1}$ and $\alpha_{2}$ describe the dipolequadrupole interference,

$$
\begin{aligned}
& \alpha_{1}=2 \operatorname{Re}\left\{A_{d}^{*}\left[g_{1}\left(\hat{\boldsymbol{\epsilon}} \cdot \hat{\mathbf{p}}_{1}\right)+g_{s}\left(\hat{\boldsymbol{\epsilon}} \cdot \hat{\boldsymbol{p}}_{2}\right)\right]\right\}, \\
& \alpha_{2}=2 \operatorname{Re}\left\{A_{d}^{*}\left[g_{2}\left(\hat{\boldsymbol{\epsilon}} \cdot \hat{\mathbf{p}}_{2}\right)+g_{s}\left(\hat{\boldsymbol{\epsilon}} \cdot \hat{\boldsymbol{p}}_{1}\right)\right]\right\} .
\end{aligned}
$$

As noted above, the quadrupole terms in Eq. (27) do not contribute to the TDCS for the perpendicular plane geometry. Thus, the dipole TDCS (i.e., $\sigma_{d}$ ) may be measured by detecting photoelectrons in the plane perpendicular to the photon beam. The parameters $\alpha_{1}$ and $\alpha_{2}$ may be determined from two measurements employing a nonorthogonal geometry with different directions of the photon wave vector. As seen from Eqs. (27) and (28), the interference between the dipole and quadrupole TDCS contributions is strongest for the case of coplanar geometry, i.e., when the vectors $\hat{\boldsymbol{\epsilon}}, \mathbf{k}$, $\mathbf{p}_{1}$, and $\mathbf{p}_{2}$ lie in one plane (see Fig. 1). In particular, the difference between two TDCS measurements, induced by retardation corrections, is maximal when the second measurement is made with the direction of the photon beam inverted [33].

\section{Elliptic and circular polarizations}

For the most general case of DPI by elliptically polarized light described by the complex polarization vector $\mathbf{e}$, the TDCS in Eq. (13) (neglecting the small terms $\sim\left|A_{q}\right|^{2}$ ) has a model-independent parametrization similar to that for dipole DPI [24],

$$
\begin{aligned}
\sigma= & \mathcal{A}\left(c_{1}\left|\mathbf{e} \cdot \hat{\mathbf{p}}_{1}\right|^{2}+c_{2}\left|\mathbf{e} \cdot \hat{\mathbf{p}}_{2}\right|^{2}+\operatorname{Re} c_{3}\left\{( 1 - l ) \left[\left(\hat{\mathbf{p}}_{1} \cdot \hat{\mathbf{p}}_{2}\right)\right.\right.\right. \\
& \left.\left.-\left(\hat{\mathbf{k}} \cdot \hat{\mathbf{p}}_{1}\right)\left(\hat{\mathbf{k}} \cdot \hat{\mathbf{p}}_{2}\right)\right]+2 l\left(\hat{\boldsymbol{\epsilon}} \cdot \hat{\mathbf{p}}_{1}\right)\left(\hat{\boldsymbol{\epsilon}} \cdot \hat{\mathbf{p}}_{2}\right)\right\} \\
& \left.+\xi \operatorname{Im} c_{3}\left(\hat{\mathbf{k}} \cdot\left[\hat{\mathbf{p}}_{1} \times \hat{\mathbf{p}}_{2}\right]\right)\right),
\end{aligned}
$$

where, however, the coefficients $c_{i}$ are now k-dependent, 


$$
\begin{gathered}
c_{1}=\left|f_{1}\right|^{2}+2 \operatorname{Re}\left[f_{1} g_{1}^{*}\left(\hat{\mathbf{k}} \cdot \hat{\mathbf{p}}_{1}\right)+f_{1} g_{s}^{*}\left(\hat{\mathbf{k}} \cdot \hat{\mathbf{p}}_{2}\right)\right], \\
c_{2}=\left|f_{2}\right|^{2}+2 \operatorname{Re}\left[f_{2} g_{2}^{*}\left(\hat{\mathbf{k}} \cdot \hat{\mathbf{p}}_{2}\right)+f_{2} g_{s}^{*}\left(\hat{\mathbf{k}} \cdot \hat{\mathbf{p}}_{1}\right)\right], \\
c_{3}=f_{1} f_{2}^{*}+\left(f_{1} g_{s}^{*}+f_{2}^{*} g_{1}\right)\left(\hat{\mathbf{k}} \cdot \hat{\mathbf{p}}_{1}\right)+\left(f_{2}^{*} g_{s}+f_{1} g_{2}^{*}\right)\left(\hat{\mathbf{k}} \cdot \hat{\mathbf{p}}_{2}\right) .
\end{gathered}
$$

The parameter $l$ in Eq. (29) is the degree of linear polarization of an elliptically polarized photon, $l=\mathbf{e}^{2}=\sqrt{1-\xi^{2}}$, and the unit vector $\hat{\boldsymbol{\epsilon}}$ is directed along the major axis of the polarization ellipse. In dealing with the scalar products involving the complex vector $\mathbf{e}$, the following relation is convenient [24]:

$$
2|\mathbf{e} \cdot \mathbf{a}|^{2}=2 l|\hat{\boldsymbol{\epsilon}} \cdot \mathbf{a}|^{2}+(1-l)[\hat{\mathbf{k}} \times \mathbf{a}]^{2},
$$

where a is a real vector. Note also that Eq. (29) may be rewritten also in terms of the Stokes parameters $S_{j}[10]$; in particular, $\xi \equiv i\left(\hat{\mathbf{k}} \cdot\left[\mathbf{e} \times \mathbf{e}^{*}\right]\right)=-S_{3}$. The photon polarization dependence of the dipole-quadrupole TDCS is thus determined by four real $\hat{\mathbf{k}}$-dependent parameters: $c_{1}, c_{2}, \operatorname{Re} c_{3}$, and $\operatorname{Im} c_{3}$. These parameters may be determined from four measurements with different polarizations of the photon beam, e.g., two experiments with linearly polarized photons and two experiments with circularly polarized photons. Once the parameters $c_{i}$ have been determined, the TDCS corresponding to an arbitrarily polarized photon is given by Eq. (29). Thus, as for the EDA case, all information on the nondipole TDCS for an elliptic polarization may be obtained from measurements with linearly and circularly polarized light. We note that the TDCS parametrization in Eq. (29) is independent of the dynamical model used to calculate the parameters $f_{1,2}, g_{1,2}$, and $g_{s}$.

For circularly polarized photons $(l=0, \xi= \pm 1)$, Eq. (29) simplifies [taking into account Eq. (31)],

$$
\begin{aligned}
\sigma= & \frac{\mathcal{A}}{2}\left\{c_{1}\left[\hat{\mathbf{k}} \times \hat{\mathbf{p}}_{1}\right]^{2}+c_{2}\left[\hat{\mathbf{k}} \times \hat{\mathbf{p}}_{2}\right]^{2}\right. \\
& +\operatorname{Re} c_{3}\left(\left[\hat{\mathbf{k}} \times \hat{\mathbf{p}}_{1}\right] \cdot\left[\hat{\mathbf{k}} \times \hat{\mathbf{p}}_{2}\right]\right) \\
& \left.+2 \xi \operatorname{Im} c_{3}\left(\hat{\mathbf{k}} \cdot\left[\hat{\mathbf{p}}_{1} \times \hat{\mathbf{p}}_{2}\right]\right)\right\} .
\end{aligned}
$$

The TDCSs in Eqs. (29) and (32) both contain a term that is proportional to the degree of circular polarization $\xi$; this term is sensitive to the sign of $\xi$, i.e., it is responsible for the CD effect. This effect is usually characterized by the absolute CD parameter, $\Delta_{\mathrm{CD}} \equiv \sigma(\xi=+1)-\sigma(\xi=-1)$, which has the following form:

$$
\Delta_{\mathrm{CD}}=2 \mathcal{A} \operatorname{Im} c_{3}\left(\hat{\mathbf{k}} \cdot\left[\hat{\mathbf{p}}_{1} \times \hat{\mathbf{p}}_{2}\right]\right) .
$$

The term $\Delta_{\mathrm{CD}}$ involves both the E1-E1 and E1-E2 contributions,

$$
\begin{gathered}
\Delta_{\mathrm{CD}}=\Delta_{\mathrm{CD}}^{(d i p)}+\Delta_{\mathrm{CD}}^{(\text {quadr })}, \\
\Delta_{\mathrm{CD}}^{(d i p)}=2 \mathcal{A} \operatorname{Im}\left(f_{1} f_{2}^{*}\right)\left(\hat{\mathbf{k}} \cdot\left[\hat{\mathbf{p}}_{1} \times \hat{\mathbf{p}}_{2}\right]\right),
\end{gathered}
$$

$$
\begin{aligned}
\Delta_{\mathrm{CD}}^{(\text {quadr })}= & 2 \mathcal{A} \operatorname{Im}\left[\left(f_{1} g_{s}^{*}+f_{2}^{*} g_{1}\right)\left(\hat{\mathbf{k}} \cdot \hat{\mathbf{p}}_{1}\right)\right. \\
& \left.+\left(f_{2}^{*} g_{s}+f_{1} g_{2}^{*}\right)\left(\hat{\mathbf{k}} \cdot \hat{\mathbf{p}}_{2}\right)\right]\left(\hat{\mathbf{k}} \cdot\left[\hat{\mathbf{p}}_{1} \times \hat{\mathbf{p}}_{2}\right]\right),
\end{aligned}
$$

which have different symmetry properties with respect to the inversion of the photon beam (i.e., $\mathbf{k} \rightarrow-\mathbf{k}$ ), and with respect to reflection of the photoelectron pair in the polarization plane [i.e., $\left(\theta_{1}, \theta_{2}\right) \rightarrow\left(\pi-\theta_{1}, \pi-\theta_{2}\right)$, where $\theta_{1,2}$ are the spherical angles of the vectors $\hat{\mathbf{p}}_{1,2}$ in the coordinate system whose $z$ axis is directed along the photon wave vector $\mathbf{k}$ ]. Within the EDA framework, the CD effect is described by the dipole term $\Delta_{\mathrm{CD}}^{(\text {dip })}[20,24]$, which vanishes at equal energy sharing (because for $p_{1}=p_{2}$ one has $f_{1}=f_{2}$ ). However, the quadrupole term, $\Delta_{\mathrm{CD}}^{\text {(quadr) }}$, produces a nonzero $\mathrm{CD}$ effect even at equal energy sharing. Note that the imaginary part of $c_{3}$ in Eq. (33) changes sign upon making the substitutions $\mathbf{p}_{1} \rightleftarrows \mathbf{p}_{2}$, as it should because of the invariance of the total CD term, $\Delta_{\mathrm{CD}}$, upon interchange of photoelectrons.

The dichroic term $\operatorname{Im} c_{3}\left(\hat{\mathbf{k}} \cdot\left[\hat{\mathbf{p}}_{1} \times \hat{\mathbf{p}}_{2}\right]\right)$ in Eqs. (29) and (32) results also in an unusual feature of the TDCS, the different symmetry of the TDCS with respect to two transformations: (i) $\mathbf{k} \rightarrow-\mathbf{k}$ (i.e., the inversion of the photon beam direction) and (ii) $\left(\theta_{1}, \theta_{2}\right) \rightarrow\left(\pi-\theta_{1}, \pi-\theta_{2}\right)$ (i.e., the reflection of the photoelectron pair in the polarization plane). The nonequivalence of these two transformations, i.e., the fact that $\sigma\left(-\mathbf{k}, \theta_{1}, \theta_{2}, \xi\right) \neq \sigma\left(\mathbf{k}, \pi-\theta_{1}, \pi-\theta_{2}, \xi\right)$, may be verified by simple inspection of Eqs. (29) and (32). Each of the terms in these equations, except for the term $2 \mathcal{A} \operatorname{Im} c_{3}\left(\hat{\mathbf{k}} \cdot\left[\hat{\mathbf{p}}_{1} \times \hat{\mathbf{p}}_{2}\right]\right)$, transforms in the same way upon either of the two transformations. On the contrary, the term $2 \mathcal{A} \operatorname{Im} c_{3}\left(\hat{\mathbf{k}} \cdot\left[\hat{\mathbf{p}}_{1} \times \hat{\mathbf{p}}_{2}\right]\right)$ transforms differently: the scalar product $\left(\hat{\mathbf{k}} \cdot\left[\hat{\mathbf{p}}_{1} \times \hat{\mathbf{p}}_{2}\right]\right)$ changes its sign when $\mathbf{k} \rightarrow-\mathbf{k}$, but is invariant with respect to $\left(\theta_{1}, \theta_{2}\right) \rightarrow\left(\pi-\theta_{1}, \pi-\theta_{2}\right)$. From the considerations above, the following relation follows:

$$
\sigma\left(\mathbf{k}, \theta_{1}, \theta_{2}, \xi\right)=\sigma\left(-\mathbf{k}, \pi-\theta_{1}, \pi-\theta_{2},-\xi\right),
$$

which reflects the invariance of the TDCS upon spatial inversion (taking into account that $\xi$ is a pseudoscalar quantity) and shows that both transformations (i) and (ii) are equivalent in the limit $l \rightarrow 1$ (linear polarization). The difference between the TDCSs resulting from the transformations (i) and (ii) is

$$
\sigma\left(-\mathbf{k}, \theta_{1}, \theta_{2}, \xi\right)-\sigma\left(\mathbf{k}, \pi-\theta_{1}, \pi-\theta_{2}, \xi\right)=\xi\left(\Delta_{\mathrm{CD}}^{(q u a d r)}-\Delta_{\mathrm{CD}}^{(d i p)}\right)
$$

The retardation-induced part of this difference, $\xi \Delta_{\mathrm{CD}}^{(\text {quadr })}$, may be observed most easily at equal energy sharing, when the generally large dipole term $\Delta_{\mathrm{CD}}^{(d i p)}$ in Eq. (36) vanishes. In this case, the term $\Delta_{\mathrm{CD}}$ in Eq. (33) changes its sign when $\left(\theta_{1}, \theta_{2}\right) \rightarrow\left(\pi-\theta_{1}, \pi-\theta_{2}\right)$ but is invariant with respect to $\mathbf{k}$ $\rightarrow-\mathbf{k}$. We note that besides the case of linear polarization, the asymmetries of the TDCS resulting from the transformations (i) and (ii) become equivalent also for geometrical configurations in which the term $\Delta_{\mathrm{CD}}$ vanishes, e.g., when the vectors $\hat{\mathbf{p}}_{1}, \hat{\mathbf{p}}_{2}$, and $\mathbf{k}$ lie in one plane $\left[\right.$ or when $\mathbf{k} \cdot\left(\mathbf{p}_{1}-\mathbf{p}_{2}\right)$ $=0$ for the case of equal energy sharing]. 


\section{Parametrizations in terms of the symmetrized amplitudes}

The expression for the TDCS in terms of symmetrized amplitudes has a form identical to that in Eqs. (29) and (30) [as well as to those in Eqs. (32)-(34)] provided the following substitutions are made: $\left\{\hat{\mathbf{p}}_{1}, \hat{\mathbf{p}}_{2}, f_{1}, f_{2}, g_{1}, g_{2}, g_{s}\right\}$ $\rightarrow\left\{\mathbf{p}_{+}, \mathbf{p}_{-}, f^{(\mathrm{g})}, f^{(\mathrm{u})}, g_{+}^{(\mathrm{g})}, g_{-}^{(\mathrm{g})}, g^{(\mathrm{u})}\right\}$. Thus in general this parametrization does not simplify the analysis [because only the term corresponding to $\left(\mathbf{p}_{1} \cdot \mathbf{p}_{2}\right)$ in the second line of Eq. (29) vanishes in the symmetrized parametrization, since $\left(\mathbf{p}_{+} \cdot \mathbf{p}_{-}\right)=0$ ], however it does lead to a simpler form of the TDCS in particular cases.

The TDCS has an especially simple form for back-to-back electron emission (i.e., $\mathbf{p}_{2}=-\mathbf{p}_{1}$ or $\mathbf{p}_{+}=0$ ),

$$
\sigma=\mathcal{A}\left\{\left|f^{(u)}\right|^{2}+2 \operatorname{Re}\left[f^{(u)} g_{-}^{(\mathrm{g})^{*}}\right]\left(\hat{\mathbf{k}} \cdot \mathbf{p}_{1}\right)\right\}\left|\mathbf{e} \cdot \mathbf{p}_{1}\right|^{2} .
$$

If the wave vector $\mathbf{k}$, photoelectron momenta $\mathbf{p}_{1}=-\mathbf{p}_{2}$, and the major axis of the polarization ellipse lie in the same plane, the forward-backward asymmetry, $\Delta=\sigma(\mathbf{e}, \mathbf{k})-\sigma(\mathbf{e}$, $\mathbf{- k}$ ), for this case may be analyzed as a function of the angle $\varphi_{\mathbf{k}}$ between the wave vector $\mathbf{k}$ and the photoelectron ejection axis directed along $\mathbf{p}_{1}=-\mathbf{p}_{2}$ [owing to Eq. (31)],

$$
\Delta=2 \mathcal{A}(1+l) \operatorname{Re}\left\{f^{(u)} g_{-}^{(\mathrm{g}) *}\right\} \sin ^{2} \varphi_{\mathbf{k}} \cos \varphi_{\mathbf{k}} .
$$

This asymmetry may be visible at energy sharings close to equal, in which case $f^{(u)}$ vanishes; see the numerical results in Ref. [33].

For the case of equal energy sharing, the parametrization of the TDCS for an elliptic polarization is

$$
\begin{aligned}
\sigma^{(e q)}= & \mathcal{A}\left\{\left[\left|f^{(\mathrm{g})}\right|^{2}+2 \operatorname{Re}\left\{f^{(\mathrm{g})^{*}} g_{+}^{(\mathrm{g})}\right\}\left(\hat{\mathbf{k}} \cdot \mathbf{p}_{+}\right)\right]\left|\mathbf{e} \cdot \mathbf{p}_{+}\right|^{2}\right. \\
& +\operatorname{Re}\left\{f^{(\mathrm{g})^{*}} g_{-}^{(\mathrm{g})}\right\}\left(\hat{\mathbf{k}} \cdot \mathbf{p}_{-}\right)\left[2 l\left(\hat{\boldsymbol{\epsilon}} \cdot \mathbf{p}_{+}\right)\left(\hat{\boldsymbol{\epsilon}} \cdot \mathbf{p}_{-}\right)\right. \\
& \left.\left.+(l-1)\left(\hat{\mathbf{k}} \cdot \mathbf{p}_{+}\right)\left(\hat{\mathbf{k}} \cdot \mathbf{p}_{-}\right)\right]\right\}+(\xi / 2) \Delta_{\mathrm{CD}}^{(e q)},
\end{aligned}
$$

where $\left.\Delta_{\mathrm{CD}}^{(e q)} \equiv \Delta_{\mathrm{CD}}^{(\text {quadr })}\right|_{p_{1}=p_{2}}$; it has the following form in terms of the symmetrized amplitudes:

$$
\Delta_{\mathrm{CD}}^{(e q)}=2 \mathcal{A} \operatorname{Im}\left\{f^{(\mathrm{g})^{*}} g_{-}^{(\mathrm{g})}\right\}\left(\hat{\mathbf{k}} \cdot\left[\mathbf{p}_{-} \times \mathbf{p}_{+}\right]\right)\left(\hat{\mathbf{k}} \cdot \mathbf{p}_{-}\right) .
$$

Note that this form of the nondipole CD parameter for $p_{1}$ $=p_{2}$ in terms of the vectors $\mathbf{p}_{ \pm}$is expected since $\Delta_{\mathrm{CD}}^{(e q)}$ should be invariant with respect to the substitution $\mathbf{p}_{1} \rightleftarrows \mathbf{p}_{2}$ (i.e., $\mathbf{p}_{-} \rightarrow-\mathbf{p}_{-}$).

The "kinematical" maxima of $\Delta_{\mathrm{CD}}^{(e q)}$ may be deduced by supposing that the vectors $\mathbf{p}_{-}$and $\mathbf{p}_{+}$are directed along the $z$ and $x$ axes of a coordinate frame, so that the $y$ axis is directed along the vector product $\left[\mathbf{p}_{-} \times \mathbf{p}_{+}\right]=\left[\hat{\mathbf{p}}_{1} \times \hat{\mathbf{p}}_{2}\right] / 2[\mathrm{see}$ Fig. 2(a) ]. In terms of $\theta_{\mathbf{k}}$ and $\phi_{\mathbf{k}}$, the spherical angles of the vector $\hat{\mathbf{k}}$, we obtain

$$
\begin{aligned}
2\left(\hat{\mathbf{k}} \cdot \mathbf{p}_{-}\right)\left(\hat{\mathbf{k}} \cdot\left[\mathbf{p}_{-} \times \mathbf{p}_{+}\right]\right) & =(\hat{\mathbf{k}} \cdot \hat{\mathbf{z}})(\hat{\mathbf{k}} \cdot \hat{\mathbf{y}}) \sin (\theta / 2) \sin \theta \\
& =\sin \left(2 \theta_{\mathbf{k}}\right) \sin \phi_{\mathbf{k}} \sin ^{2}(\theta / 2) \cos (\theta / 2) .
\end{aligned}
$$

The modulus of this expression is maximal for $\theta_{\mathbf{k}}= \pm \pi / 4$ and $\phi_{\mathbf{k}}= \pm \pi / 2$ [see Fig. 2(a)]. These angles correspond to maxima of the equal energy sharing $\mathrm{CD}$ effect.
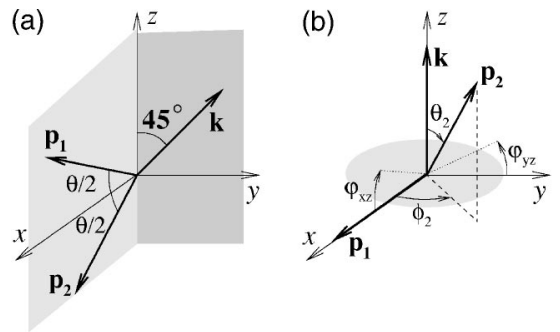

FIG. 2. Geometries suitable for observation of retardationinduced light polarization effects. (a) Geometry at which the equal energy sharing $\mathrm{CD}$ is maximal. Electrons are detected in the $x z$ plane, at angles of $\pm \theta / 2$ with respect to the $x$ axis. The photon wave vector $\mathbf{k}$ lies in the $y z$ plane and makes an angle $45^{\circ}$ with the $z$ axis. (b) Geometry for observation of retardation-induced asymmetries in the TDCS. The first electron is ejected along the $x$ axis and the second one (whose angular distribution exhibits the asymmetry) along $\left(\theta_{2}, \phi_{2}\right)$. (Concerning the angles $\varphi_{x z}$ and $\varphi_{y z}$, see Fig. 7.)

\section{PERTURBATIVE ACCOUNT OF ELECTRON CORRELATIONS IN THE DPI QUADRUPOLE TRANSITION AMPLITUDE}

\section{A. General equations for LOPT amplitudes}

We use LOPT in the interelectron interaction to account for electron correlations in our numerical calculations of the reduced matrix elements of the operators $\mathbf{D}$ and $Q_{2 m}$ and the corresponding TDCSs (see Refs. $[14,16]$ ). The application of this approach to DPI within the EDA was described in detail in Ref. [16]; therefore, we provide here only a brief description of this approach to evaluating the quadrupole transition amplitude.

In lowest order in $1 / r_{12}$, the DPI quadrupole transition amplitude $A_{q}$ in Eq. (14) is given by

$$
A_{q}=Q_{F}\left(\mathbf{p}_{1}, \mathbf{p}_{2}\right)+Q_{F}\left(\mathbf{p}_{2}, \mathbf{p}_{1}\right)+Q_{G}\left(\mathbf{p}_{1}, \mathbf{p}_{2}\right)+Q_{G}\left(\mathbf{p}_{2}, \mathbf{p}_{1}\right),
$$

where the terms $Q_{F}$ and $Q_{G}$ account for final-state correlations (FSC) and ground-state correlations (GSC), respectively; they are shown schematically in Fig. 3. The dipole parts of the FSC and GSC terms are evaluated in Ref. [16]. The quadrupole terms, $Q_{F}$ and $Q_{G}$, have the following expressions [cf. Eqs. (7)-(9) in Ref. [16]]:

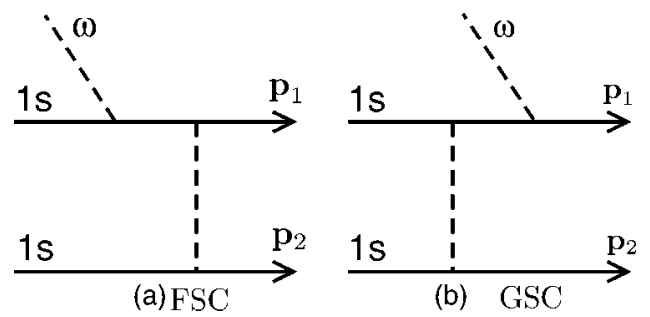

FIG. 3. Schematic diagrams for first-order perturbative contributions to the DPI amplitude. (a) Final-state correlation; (b) groundstate correlation. Two additional contributing diagrams with exchanged $\mathbf{p}_{1}$ and $\mathbf{p}_{2}$ are not shown. 


$$
\begin{gathered}
Q_{F}\left(\mathbf{p}_{1}, \mathbf{p}_{2}\right)=\sqrt{2}\left\langle\psi_{\mathbf{p}_{1}}^{(-)}\left|U_{\mathbf{p}_{2}}^{(-)}(\mathbf{r}) G_{\mathcal{E}_{F}}\left(\mathbf{r}, \mathbf{r}^{\prime}\right)\left(\mathbf{k} \cdot \mathbf{r}^{\prime}\right)\left(\mathbf{e} \cdot \nabla_{\mathbf{r}^{\prime}}\right)\right| \varphi_{0}\right\rangle, \\
Q_{G}\left(\mathbf{p}_{1}, \mathbf{p}_{2}\right)=\sqrt{2}\left\langle\psi_{\mathbf{p}_{1}}^{(-)}\left|(\mathbf{k} \cdot \mathbf{r})\left(\mathbf{e} \cdot \nabla_{\mathbf{r}}\right) G_{\mathcal{E}_{G}}\left(\mathbf{r}, \mathbf{r}^{\prime}\right) U_{\mathbf{p}_{2}}^{(-)}\left(\mathbf{r}^{\prime}\right)\right| \varphi_{0}\right\rangle, \\
U_{\mathbf{p}_{2}}^{(-)}(\mathbf{r})=\left\langle\psi_{\mathbf{p}_{2}}^{(-)}|| \mathbf{r}-\left.\mathbf{r}^{\prime}\right|^{-1} \mid \varphi_{0}\right\rangle
\end{gathered}
$$

where $G_{\mathcal{E}_{F}}$ and $G_{\mathcal{E}_{G}}$ are the one-particle Coulomb Green's functions (CGFs) with energy parameters $\mathcal{E}_{F}=E_{p_{1}}+E_{p_{2}}-\epsilon_{1 s}$ and $\mathcal{E}_{G}=2 \epsilon_{1 s}-E_{p_{2}}$; the functions $\varphi_{0}$ and $\psi_{\mathbf{p}}^{(-)}$are one-particle Coulomb wave functions for a hydrogenlike ion that describe, respectively, the ground state and continuum state normalized according to $\left\langle\psi_{\mathbf{p}} \mid \psi_{\mathbf{p}^{\prime}}\right\rangle=\delta\left(\mathbf{p}-\mathbf{p}^{\prime}\right)$; the factor $\sqrt{2}$ in Eqs. (43) and (45) accounts for symmetrization of the final state. The two exchange amplitudes are obtained by interchanging the momentum vectors $\mathbf{p}_{1}$ and $\mathbf{p}_{2}$ in Eqs. (43)-(45) and using $\mathcal{E}_{G}=2 \epsilon_{1 s}-E_{p_{1}}$.

The amplitudes $Q_{F}$ and $Q_{G}$ are evaluated by using the standard multipole expansions for the Coulomb continuum functions $\psi_{\mathbf{p}}$, for the CGF, and for $1 / r_{12}$ in Eqs. (43)-(45),

$$
\begin{gathered}
\psi_{\mathbf{p}}^{(-)}(\mathbf{r})=\frac{(2 \pi)^{-3 / 2}}{2 p} \sum_{l=0}^{\infty} i^{l}(2 l+1) \times e^{-i \delta_{l}(p)} R_{p l}(r) P_{l}(\hat{\mathbf{p}} \cdot \hat{\mathbf{r}}) \\
G_{E}\left(\mathbf{r}, \mathbf{r}^{\prime}\right)=\sum_{l, m} g_{l}\left(E ; r, r^{\prime}\right) Y_{l m}(\hat{\mathbf{r}}) Y_{l m}^{*}\left(\hat{\mathbf{r}}^{\prime}\right), \\
\frac{1}{\left|\mathbf{r}-\mathbf{r}^{\prime}\right|}=\sum_{l=0}^{\infty} \frac{r_{<}^{l}}{r_{>}^{l+1}} P_{l}\left(\hat{\mathbf{r}} \cdot \hat{\mathbf{r}}^{\prime}\right),
\end{gathered}
$$

where $\delta_{l}(p)=\arg \Gamma(l+1+i \eta)$ is a Coulomb phase shift, $\eta$ $=Z / p$, and $r_{<}=\min \left(r, r^{\prime}\right), r_{>}=\max \left(r, r^{\prime}\right) . R_{p l}(r)$ is the radial part of the Coulomb wave function,

$$
R_{p l}(r)=\frac{C_{p l}(2 p r)^{l}}{(2 l+1) !} e^{-i p r} \Phi(i \eta+l+1,2 l+2,2 i p r),
$$

where $\Phi$ is a confluent hypergeometric function and $C_{p l}$ $=2 p \exp (\pi \eta / 2)|\Gamma(l+1+i \eta)|$. The radial function (49) is normalized as follows: $\int_{0}^{\infty} R_{p l}(r) R_{p^{\prime} l}(r) r^{2} d r=2 \pi \delta\left(p-p^{\prime}\right)$. For the radial part of the CGF, we use the integral representation [38],

$$
\begin{aligned}
g_{l}\left(E ; r, r^{\prime}\right)= & \frac{2}{\sqrt{r r^{\prime}}} \int_{0}^{1} \frac{d u}{1-u} u^{-Z \nu-1 / 2} \\
& \times \exp \left\{-\frac{r+r^{\prime}}{\nu} \frac{1+u}{1-u}\right\} I_{2 l+1} \\
& \times\left(\frac{4 \sqrt{r r^{\prime} u}}{\nu(1-u)}\right),
\end{aligned}
$$

where $\nu=1 / \sqrt{-2 E}(\nu=i|\nu|$ for $E>0)$ and $I_{n}(x)$ is a modified Bessel function.

\section{B. Evaluation of angular integrals}

As a result of the partial wave expansions in Eqs. (46)-(48), the quadrupole amplitudes $Q_{F, G}$ in Eqs. (43) and (44) take the form of an infinite double sum over the electron angular momenta, $l_{1}$ and $l_{2}$, that characterize the corresponding partial wave amplitudes. We consider here the evaluation of the angular integrals in these partial wave amplitudes. The angular integral over $\hat{\mathbf{r}}$ for the $\left(l, l^{\prime}\right)$ partial wave amplitude $Q_{F}$ in Eq. (43) has the form

$$
\begin{aligned}
I_{F}^{\left(l, l^{\prime}\right)}= & \int P_{l}\left(\hat{\mathbf{p}}_{1} \cdot \hat{\mathbf{r}}\right) P_{l^{\prime}}\left(\hat{\mathbf{p}}_{2} \cdot \hat{\mathbf{r}}\right)(\hat{\mathbf{k}} \cdot \hat{\mathbf{r}})(\mathbf{e} \cdot \hat{\mathbf{r}}) d \hat{\mathbf{r}} \\
& =C_{I}^{\left(l, l^{\prime}\right)}\left(\{\hat{\mathbf{k}} \otimes \mathbf{e}\}_{2} \cdot \mathcal{C}_{2}^{l l^{\prime}}\left(\hat{\mathbf{p}}_{1}, \hat{\mathbf{p}}_{2}\right)\right),
\end{aligned}
$$

where

$$
C_{I}^{\left(l, l^{\prime}\right)}=\frac{4 \pi}{5} \sqrt{\frac{2}{3}} C_{l 0 l^{\prime} 0}^{20} .
$$

The scalar product of the rank-2 tensors in Eq. (52) may be evaluated using Eqs. (C3) and (C5) in Ref. [24],

$$
\left(\{\hat{\mathbf{k}} \otimes \mathbf{e}\}_{2} \cdot \mathcal{C}_{2}^{l l^{\prime}}\left(\hat{\mathbf{p}}_{1}, \hat{\mathbf{p}}_{2}\right)\right)=(-1)^{l} C_{S}^{\left(l, l^{\prime}\right)} A^{\left(l, l^{\prime}\right)}\left(\hat{\mathbf{p}}_{1}, \hat{\mathbf{p}}_{2}, \hat{\mathbf{k}}, \mathbf{e}\right),
$$

where

$$
\begin{aligned}
& A^{(l, l)}\left(\hat{\mathbf{p}}, \hat{\mathbf{p}}^{\prime}, \hat{\mathbf{k}}, \mathbf{e}\right)=\sqrt{6}\left\{\left[(\mathbf{e} \cdot \hat{\mathbf{p}})(\hat{\mathbf{k}} \cdot \hat{\mathbf{p}})+\left(\mathbf{e} \cdot \hat{\mathbf{p}}^{\prime}\right)\left(\hat{\mathbf{k}} \cdot \hat{\mathbf{p}}^{\prime}\right)\right] P_{l}^{(2)}(x)\right. \\
& -\left[(\mathbf{e} \cdot \hat{\mathbf{p}})\left(\hat{\mathbf{k}} \cdot \hat{\mathbf{p}}^{\prime}\right)+\left(\mathbf{e} \cdot \hat{\mathbf{p}}^{\prime}\right)(\hat{\mathbf{k}} \cdot \hat{\mathbf{p}})\right] \\
& \left.\times\left[\frac{1}{2} P_{l}^{(1)}(x)+x P_{l}^{(2)}(x)\right]\right\}, \\
& A^{\left(l, l^{\prime}=l \pm 2\right)}\left(\hat{\mathbf{p}}, \hat{\mathbf{p}}^{\prime}, \hat{\mathbf{k}}, \mathbf{e}\right)=(\mathbf{e} \cdot \hat{\mathbf{p}})(\hat{\mathbf{k}} \cdot \hat{\mathbf{p}}) P_{l}^{(2)}(x) \\
& +\left(\mathbf{e} \cdot \hat{\mathbf{p}}^{\prime}\right)\left(\hat{\mathbf{k}} \cdot \hat{\mathbf{p}}^{\prime}\right) P_{l^{\prime}}^{(2)}(x) \\
& -\left[(\mathbf{e} \cdot \hat{\mathbf{p}})\left(\hat{\mathbf{k}} \cdot \hat{\mathbf{p}}^{\prime}\right)+\left(\mathbf{e} \cdot \hat{\mathbf{p}}^{\prime}\right)\right. \\
& \times(\hat{\mathbf{k}} \cdot \hat{\mathbf{p}})] P_{\left(l+l^{\prime}\right) / 2}^{(2)}(x), \\
& C_{S}^{\left(l, l^{\prime}\right)}=\sqrt{\frac{20\left(2 l_{>}-4\right) !}{\left(2 l_{>}+1\right) !}},
\end{aligned}
$$

and where $x=\cos \theta=\left(\hat{\mathbf{p}} \cdot \hat{\mathbf{p}}^{\prime}\right)$ and $l_{>}=\max \left(l, l^{\prime}\right)$. The evaluation of the angular integrals for the $(l, l)$ partial wave amplitude $Q_{G}$ in Eq. (44) is slightly more involved. After integration over $\hat{\mathbf{r}}^{\prime}$ in Eq. (44), the remaining angular integral over $\hat{\mathbf{r}}$ in that equation has the form

$$
I_{G}^{\left(l, l^{\prime}\right)}=\int P_{l}\left(\hat{\mathbf{p}}_{1} \cdot \hat{\mathbf{r}}\right)(\hat{\mathbf{k}} \cdot \hat{\mathbf{r}})(\mathbf{e} \cdot \boldsymbol{\nabla}) P_{l^{\prime}}\left(\hat{\mathbf{p}}_{2} \cdot \hat{\mathbf{r}}\right) d \hat{\mathbf{r}},
$$

where $l^{\prime}=l, l \pm 2$. It is evaluated using the fact that $\boldsymbol{\nabla}$ $=(\partial / \partial r) \hat{\mathbf{r}}+(1 / r) \boldsymbol{\nabla}_{\Omega}$. The integral involving $\hat{\mathbf{r}}$ reduces to $I_{F}^{\left(l, l^{\prime}\right)}$; the integral involving $\boldsymbol{\nabla}_{\Omega}$ is evaluated by using the Wigner-Eckart theorem for the matrix element of the direct product of the spherical components $r_{\mu}\left(\nabla_{\Omega}\right)_{\nu}$ [36]. The resulting expression for $I_{G}^{\left(l, l^{\prime}\right)}$ is 


$$
I_{G}^{\left(l, l^{\prime}\right)}=I_{F}^{\left(l, l^{\prime}\right)} \frac{\partial}{\partial r}+J^{\left(l, l^{\prime}\right)} \frac{1}{r},
$$

where

$$
\begin{aligned}
J^{\left(l, l^{\prime}\right)} & =\int P_{l}\left(\hat{\mathbf{p}}_{1} \cdot \hat{\mathbf{r}}\right)(\hat{\mathbf{k}} \cdot \hat{\mathbf{r}})\left(\mathbf{e} \cdot \nabla_{\Omega}\right) P_{l^{\prime}}\left(\hat{\mathbf{p}}_{2} \cdot \hat{\mathbf{r}}\right) d \hat{\mathbf{r}} \\
& =C_{J}^{\left(l, l^{\prime}\right)}\left(\{\hat{\mathbf{k}} \otimes \mathbf{e}\}_{2} \cdot \mathcal{C}_{2}^{l l^{\prime}}\left(\hat{\mathbf{p}}_{1}, \hat{\mathbf{p}}_{2}\right)\right),
\end{aligned}
$$

and where $C_{J}^{\left(l, l^{\prime}\right)}$ may be expressed in terms of $C_{I}^{\left(l, l^{\prime}\right)}: C_{J}^{\left(l, l^{\prime}\right)}$ $=\left[\delta_{l^{\prime} l}(3 / 2)+\delta_{l^{\prime} l+2}\left(l^{\prime}+1\right)-\delta_{l^{\prime} l-2} l^{\prime}\right] C_{I}^{\left(l, l^{\prime}\right)}$. Thus the angular integral over $\hat{\mathbf{r}}$ in Eq. (44) becomes

$$
\begin{aligned}
I_{G}^{\left(l, l^{\prime}\right)}= & \left(\{\hat{\mathbf{k}} \otimes \mathbf{e}\}_{2} \cdot \mathcal{C}_{2}^{l l^{\prime}}\left(\hat{\mathbf{p}}_{1}, \hat{\mathbf{p}}_{2}\right)\right) \\
& \times C_{I}^{\left(l, l^{\prime}\right)}\left[\frac{\partial}{\partial r}+\frac{\delta_{l^{\prime} l}(3 / 2)+\delta_{l^{\prime} l+2}\left(l^{\prime}+1\right)-\delta_{l^{\prime} l-2} l^{\prime}}{r}\right] .
\end{aligned}
$$

Combining the above results, the partial wave expansions for the amplitudes $Q_{F, G}$ in Eqs. (43) and (44) are

$$
Q_{F, G}=\sum_{l_{1}=0}^{\infty}(-1)^{l_{1}} \sum_{l_{2}=l_{1}, l_{1} \pm 2} Q_{F, G}^{\left(l_{1}, l_{2}\right)}\left(p_{1}, p_{2}\right) \times A^{\left(l_{1}, l_{2}\right)}\left(\hat{\mathbf{p}}_{1}, \hat{\mathbf{p}}_{2}, \hat{\mathbf{k}}, \mathbf{e}\right)
$$

where the function $A^{\left(l_{1}, l_{2}\right)}\left(\hat{\mathbf{p}}_{1}, \hat{\mathbf{p}}_{2}, \hat{\mathbf{k}}, \mathbf{e}\right)[\mathrm{cf}$. Eqs. (55) and (56)] determines the polarization and angular dependence of the DPI amplitude, while $Q_{F, G}^{\left(l_{1}, l_{2}\right)}\left(p_{1}, p_{2}\right)$ is a dynamical factor that determines its energy dependence,

$$
Q_{F, G}^{\left(l_{1}, l_{2}\right)}\left(p_{1}, p_{2}\right)=\mathcal{P}_{l_{1} l_{2}} \mathcal{Q}_{F, G}^{\left(l_{1}, l_{2}\right)}\left(p_{1}, p_{2}\right),
$$

where

$$
\begin{aligned}
\mathcal{P}_{l_{1} l_{2}}= & \frac{(-1)^{l_{>}}}{(2 \pi)^{2}} \frac{\alpha \omega}{p_{1} p_{2}} e^{i\left[\delta_{l_{1}}\left(p_{1}\right)+\delta_{l_{2}}\left(p_{2}\right)\right]} \\
& \times\left(2 l_{1}+1\right) \sqrt{\frac{4\left(2 l_{>}-4\right) !}{15\left(2 l_{>}+1\right) !}} C_{l_{1} 0 l_{2} 0}^{20},
\end{aligned}
$$

and $\mathcal{Q}_{F, G}^{\left(l_{1}, l_{2}\right)}\left(p_{1}, p_{2}\right)$ is a radial matrix element. As is shown in Appendix A, $\mathcal{Q}_{F, G}^{\left(l_{1}, l_{2}\right)}\left(p_{1}, p_{2}\right)$ may be represented as a twodimensional integral of elementary functions.

\section{LOPT results for the DPI dynamical factors}

The photon polarization and angular dependence [described by Eqs. (55), (56), and (62)] of the quadrupole part of the DPI amplitude, given (within LOPT) by Eqs. (43) and (44)], have the same form as the exact parametrization in Eqs. (21) and (22). Moreover, the equations above allow one to obtain the explicit expressions for the LOPT limit of the exact dynamical factors $Q_{l_{1} l_{2}}\left(p_{1}, p_{2}\right)$ in Eqs. (22) and (23), which involve the two-electron reduced matrix element $\left\langle p p^{\prime} ;\left(l l^{\prime}\right) 2\left\|Q_{2}\right\| 0\right\rangle$,

$$
\begin{aligned}
Q_{l_{1} l_{2}}\left(p_{1}, p_{2}\right)= & \mathcal{P}_{l_{1} l_{2}}\left[\mathcal{Q}_{F}^{\left(l_{1}, l_{2}\right)}\left(p_{1}, p_{2}\right)+\mathcal{Q}_{F}^{\left(l_{2}, l_{1}\right)}\left(p_{2}, p_{1}\right)\right. \\
& \left.+\mathcal{Q}_{G}^{\left(l_{1}, l_{2}\right)}\left(p_{1}, p_{2}\right)+\mathcal{Q}_{G}^{\left(l_{2}, l_{1}\right)}\left(p_{2}, l_{2}\right)\right]
\end{aligned}
$$

where the formulas for the radial matrix elements $\mathcal{Q}_{F, G}^{\left(l_{1}, l_{2}\right)}$ $\left(p_{1}, p_{2}\right)$ are given in Appendix A.

The LOPT analysis of dipole DPI in Ref. [16] permits one to obtain the LOPT limit of the reduced dipole matrix elements $\left\langle p p^{\prime} ;\left(l l^{\prime}\right) 1\|\mathbf{D}\| 0\right\rangle$ in the dynamical factors $D_{l_{1} l_{2}}\left(p_{1}, p_{2}\right)$ in Eq. (17). These expressions have the following form:

$$
\begin{aligned}
D_{l_{1} l_{2}}\left(p_{1}, p_{2}\right)= & \mathcal{S}_{l_{1} l_{2}}\left[\mathcal{D}_{F}^{\left(l_{1}, l_{2}\right)}\left(p_{1}, p_{2}\right)+\mathcal{D}_{F}^{\left(l_{2}, l_{1}\right)}\left(p_{2}, p_{1}\right)\right. \\
& \left.+\mathcal{D}_{G}^{\left(l_{1}, l_{2}\right)}\left(p_{1}, p_{2}\right)+\mathcal{D}_{G}^{\left(l_{2}, l_{1}\right)}\left(p_{2}, p_{1}\right)\right],
\end{aligned}
$$

where

$$
\mathcal{S}_{l_{1} l_{2}}=\frac{\sqrt{2}}{8 \pi^{2}\left(2 l_{2}+1\right) p_{1} p_{2}} e^{i\left[\delta_{l_{1}}\left(p_{1}\right)+\delta_{l_{2}}\left(p_{2}\right)\right]} .
$$

The quantities $\mathcal{D}_{F, G}^{\left(l_{1}, l_{2}\right)}\left(p_{1}, p_{2}\right)$ are the radial matrix elements of the electric-dipole operator,

$$
\begin{aligned}
& \mathcal{D}_{F}^{\left(l_{1}, l_{2}\right)}\left(p_{1}, p_{2}\right) \equiv 4 Z_{s}^{4} \mathcal{R}_{F}^{\left(l_{1}, l_{2}\right)}\left(p_{1}, p_{2}\right), \\
& \mathcal{D}_{G}^{\left(l_{1}, l_{2}\right)}\left(p_{1}, p_{2}\right) \equiv 4 Z_{s}^{3} \mathcal{R}_{G}^{\left(l_{1}, l_{2}\right)}\left(p_{1}, p_{2}\right),
\end{aligned}
$$

where the explicit expressions for $\mathcal{R}_{F, G}^{\left(l_{1}, l_{2}\right)}\left(p_{1}, p_{2}\right)$ in terms of two-dimensional integrals of elementary functions [similar to those for $\mathcal{Q}_{F, G}^{\left(l_{1}, l_{2}\right)}\left(p_{1}, p_{2}\right)$ in Appendix A] are given by Eqs. (A14), (A25), and (A28) in Ref. [16] along with their numerical LOPT values for several excess energies and energy sharings.

The dynamical factors above represent the lowest-order terms in the expansion of the exact DPI transition amplitude in $1 / Z$; they may thus be $Z$-scaled (see Refs. $[16,17]$ ) in order to treat DPI of He-like ions. Moreover, a similar scaling may be useful for estimating the DPI cross sections for the K-shells of heavy atoms, using the effective nuclear charge $Z_{e f f}=\sqrt{\left|E_{0}\right|}$ [19]. As shown in Ref. [19], such estimates for K-shell DPI are in reasonable agreement with experimental measurements of the ratio $\sigma^{++} / \sigma^{+}$for a number of atoms.

\section{NUMERICAL RESULTS AND DISCUSSIONS}

In this section we present our theoretical results, which include both dipole and quadrupole contributions to the transition amplitude, and compare them both to our EDA predictions and to experimental data, when available. As discussed in Sec. II C, there are two manifestations of nondipole corrections: (i) two kinds of forward-backward asymmetry of the TDCS with respect to the propagation direction of the photon beam, and (ii) the dependence of the TDCS upon the sign of the degree of circular polarization (the $\mathrm{CD}$ effect) for equal energy sharing, which does not exist in the EDA. Analyses of these two kinds of nondipole effects are presented below for three representative excess energies, i.e., 20, 100, and $450 \mathrm{eV}$. (Some results for 80 and $239 \mathrm{eV}$ may be found in Refs. [33,34].) 

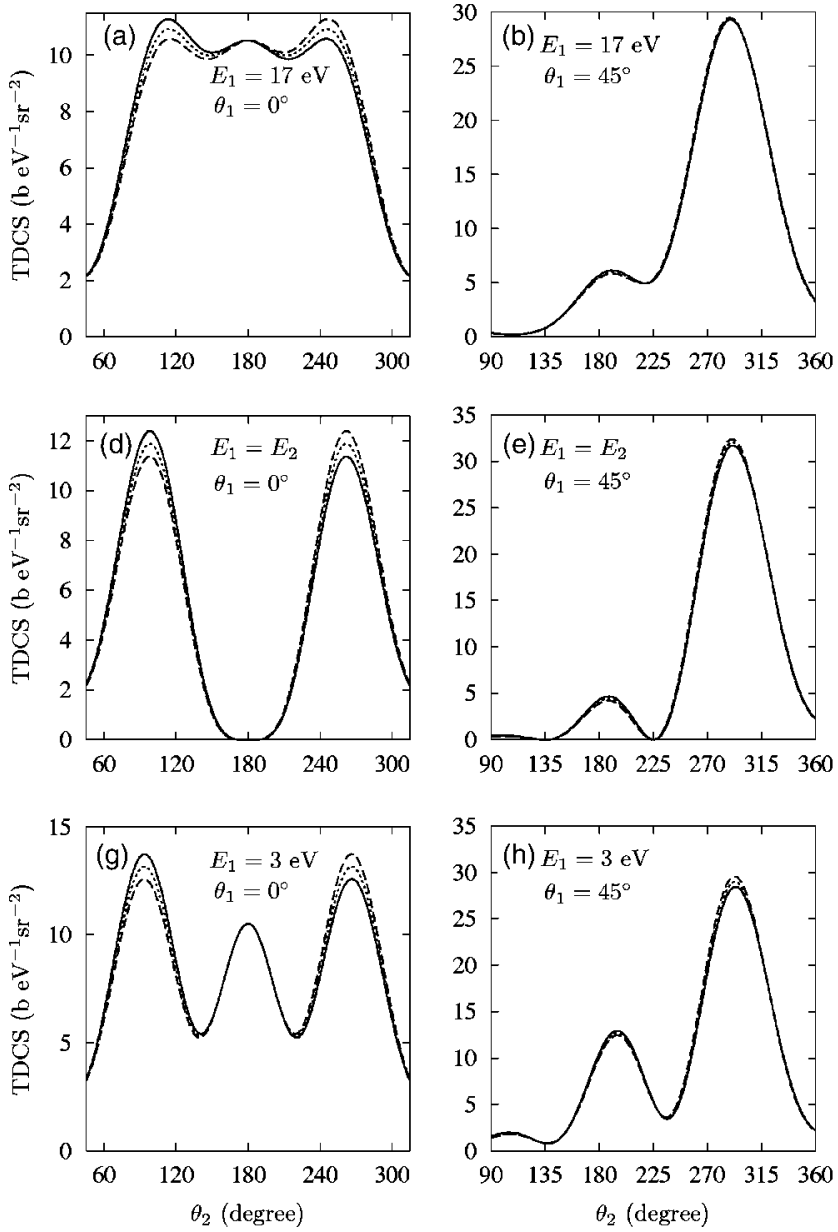

Our treatment of electron correlations at these three representative excess energies is described in Sec. III above (as well as in Refs. [14,16]). In brief, for excess energies of 20 and $100 \mathrm{eV}$, electron correlations are accounted for explicitly only in the final state, i.e., only the FS amplitude $Q_{F}$ in Eq. (43) is taken into account. Closer to the double ionization threshold, i.e., at the excess energy of $20 \mathrm{eV}$, it appears that the use of a single basis-set calculated for an effective "screening" charge $Z_{s}=Z=27 / 16$ [14] provides the most accurate agreement with both experiment and other calculations. Further away from the threshold, i.e., at $100 \mathrm{eV}$, we use the screening charge $Z_{s}=27 / 16$ only to describe approximately the He ground state [i.e., substituting $Z \rightarrow Z_{s}$ only in the hydrogenlike ground state $\left|\varphi_{0}\right\rangle$ in Eq. (43)] and take into account final-state correlations within the Coulomb basis set with $Z=2$ [16]. For an excess energy of $450 \mathrm{eV}$, electron correlations in both initial and final states [i.e., both FS and GS amplitudes $Q_{F}$ and $Q_{G}$ in Eqs. (43) and (44)] need to be taken into account, using a single Coulomb basis set evaluated for $Z=2$, thus providing gauge-independent predictions for TDCSs.

As the major goal of the present work is to examine the manifestation and importance of lowest-order nondipole effects, for consistency we compare our dipole-quadrupole results with our own EDA results (and with experimental data, when available). Note that our EDA results have already been compared with the EDA results of more sophisticated calculations elsewhere $[14,16]$.
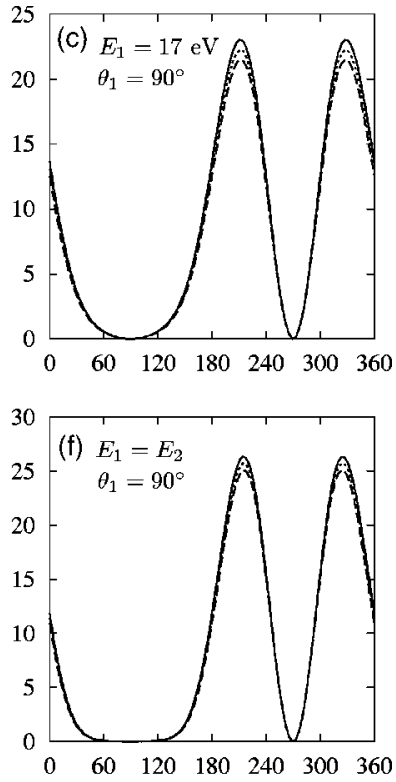

FIG. 4. Present results for the TDCS for DPI of $\mathrm{He}$ at an excess energy of $20 \mathrm{eV}$ for the case of linear polarization and coplanar geometry. Energy sharings and ejection angles are as indicated. Full curves, dipole-quadrupole results; dashed curves, dipolequadrupole results for the opposite direction of the photon beam; dotted curves, EDA results. (Note that $1.0 \mathrm{~b}=1.0 \times 10^{-24} \mathrm{~cm}^{2}$.)

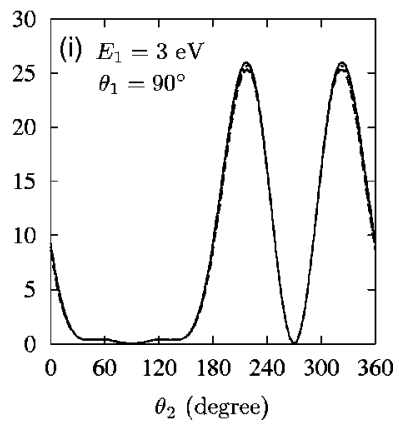

\section{A. Nondipole asymmetries in the TDCS}

In this subsection we present our numerical results for the two kinds of nondipole forward-backward asymmetry of the dipole-quadrupole TDCSs that are discussed in Sec. II C 2. For linear polarization, these two kinds of asymmetry, i.e., with respect to ejection of one of the electrons in the forward or backward directions, and with respect to inversion of the photon beam direction, are equivalent. For circular polarization, these asymmetries are in general qualitatively different and stem from different terms in the TDCS, even in the regime of equal energy sharing, which would not be the case within the EDA treatment.

\section{Excess energy of $20 \mathrm{eV}$}

The TDCSs for DPI of a He atom have been studied in great detail at an excess energy of $20 \mathrm{eV}$, both experimentally and theoretically. Absolute experimental TDCS data exist for both linear [39] and circular [32] polarization. In Fig. 4, our TDCS results for linear polarization at $20 \mathrm{eV}$ excess energy are presented for three energy sharings and three values of the ejection angle $\theta_{1}$. Because in the regime of not very asymmetric energy sharing, considered here, our model generally predicts unphysical maxima in the TDCS for small mutual ejection angles (owing to our lowest-order account of FS correlations), our predictions are given only in the angular ranges where we expect them to be accurate. In the plots for $\theta_{1}=90^{\circ}$, the geometrical zeros at $\theta_{1}=\theta_{2}=90^{\circ}$ allow us to 

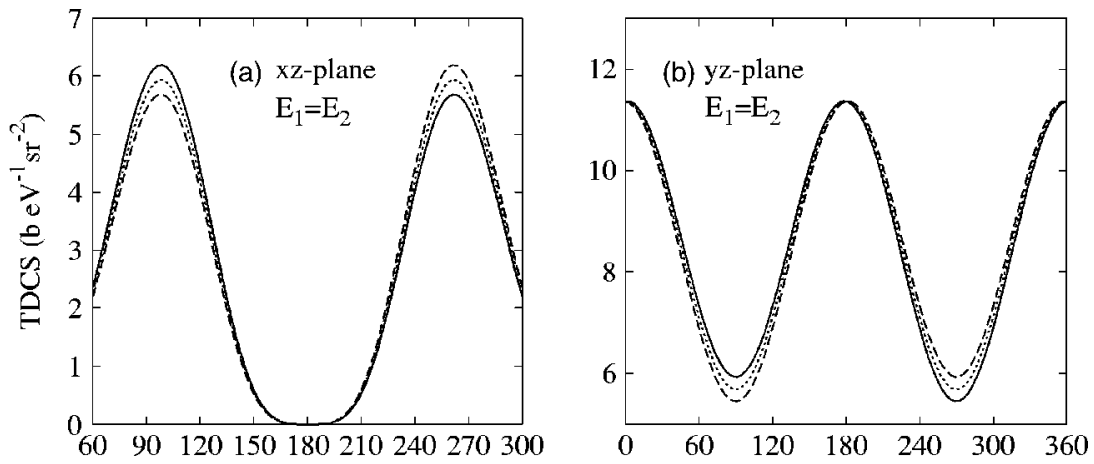

FIG. 5. Present TDCS results for DPI of He at an excess energy of $20 \mathrm{eV}$ for circular polarization and for the geometry shown in Fig. 2(b). Full curves, dipolequadrupole results; dashed curves, dipole-quadrupole results for the opposite direction of the photon beam; dotted curves, corresponding EDA results. (Note that $1.0 \mathrm{~b}$ $=1.0 \times 10^{-24} \mathrm{~cm}^{2}$.)
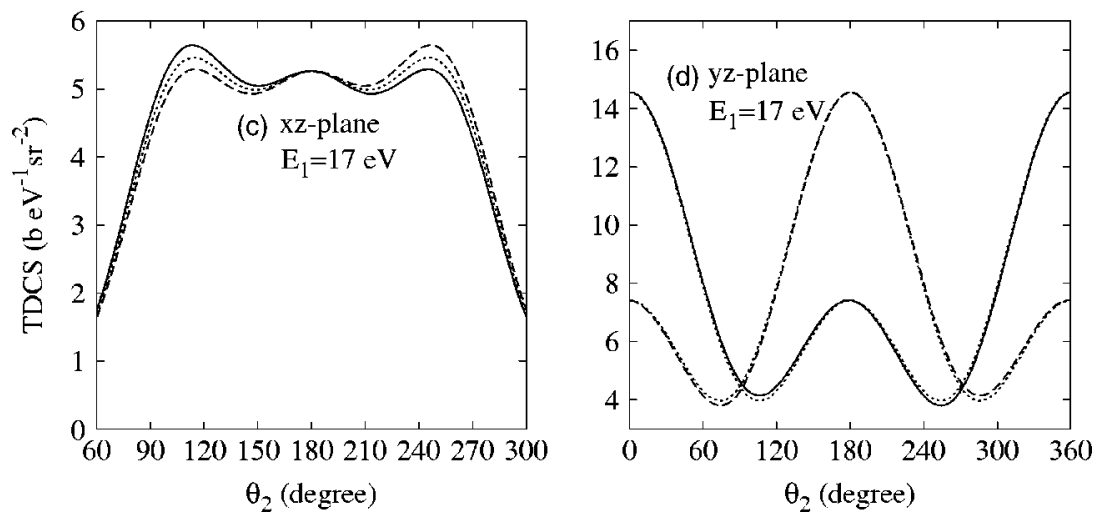

obtain accurate predictions even for small mutual ejection angles; thus for this case we present them over the full angular range.

From Fig. 4, one sees that the difference between results that account for the lowest-order retardation corrections and the EDA results is largest in absolute magnitude where the TDCSs have maxima. This occurs for the cases when one electron is ejected either at $\theta_{1}=0^{\circ}$ or $\theta_{1}=90^{\circ}$. On the contrary, for $\theta_{1}=45^{\circ}$ (and, in general, for $15 \lesssim \theta_{1} \lesssim 75^{\circ}$; data not shown) the nondipole contributions are less important. The nondipole modifications of the angular distributions appear to depend greatly upon a number of parameters, such as the ejection angle of one of the electrons, $\theta_{1}$. For $\theta_{1}=0^{\circ}$, the modification of the TDCS follows the intuitive expectation, i.e., the whole angular distribution is shifted in the "forward" direction [Figs. 4(a), 4(d), and 4(g)]. For $\theta_{1}=90^{\circ}$, however, the magnitude of the TDCS is increased by nondipole corrections in the region of the two TDCS maxima, which both lie in the "backward" half-plane [Figs. 4(c), 4(f), and 4(i)].

Our predictions for DPI by circularly polarized light (for the case $\xi=+1)$ are shown in Fig. 5 for the cases of equal and unequal energy sharings. The electron having energy $E_{1}$ is ejected along the $x$ axis. The first nondipole feature of the TDCS, which appears for both equal and unequal energy sharings, is the forward-backward asymmetry (i.e., with respect to inversion of the photon beam propagation direction), which is similar to that for the case of linear polarization. The second nondipole feature is the nonzero CD effect for the case of equal energy sharing. In the case when the second electron is detected in the $x z$ plane [Figs. 5(a) and 5(c)], neither the dipole nor the nondipole CD terms in Eq. (39) contribute, and the TDCS exhibits the following property: the TDCS in the angular range $180^{\circ}<\theta_{2}<360^{\circ}$ equals the
TDCS in the angular range $0^{\circ}<\theta_{2}<180^{\circ}$ for a photon beam propagating in the opposite direction, i.e., the outcomes of these two measurements are equivalent. (Such a symmetry is also present in the case of linear polarization.) If, however, the second electron is detected in the $y z$ plane [Figs. 5(b) and $5(\mathrm{~d})$ ], the nondipole CD term in Eq. (39) contributes (while the dipole CD term remains zero), and the two measurements described above give nonequivalent results. For unequal energy sharing, the symmetry described above holds for the case when the second electron is detected in the $x z$ plane, because neither dipole nor nondipole CD terms contribute, owing to geometry; when the second electron is detected in the $y z$ plane, both dipole and nondipole CD terms do contribute, and the dipole CD term significantly alters the shape of the TDCS, making the nondipole asymmetry described above not as obvious.

\section{Excess energy of $100 \mathrm{eV}$}

The excess energy of $100 \mathrm{eV}$ represents an intermediate regime of DPI for which, however, no experimental data or theoretical predictions for the TDCS appear in the literature. In Fig. 6, we present our theoretical predictions for the dipole-quadrupole TDCS by linearly polarized light for several energy sharings. For the same reason as discussed in the previous subsection with regard to Fig. 4, i.e., due to the inaccuracy of our predictions for small mutual ejection angles when the energy sharing is not extremely asymmetric, we present our predictions in plots (a), (b), (d), and (e) only in the angular ranges for which we expect them to be accurate. In plots (c) and (f), as discussed above, our predictions should be accurate over the full angular range. They should also be accurate over the full angular range for $E_{1}=99 \mathrm{eV}$ (extremely asymmetric energy sharing). 

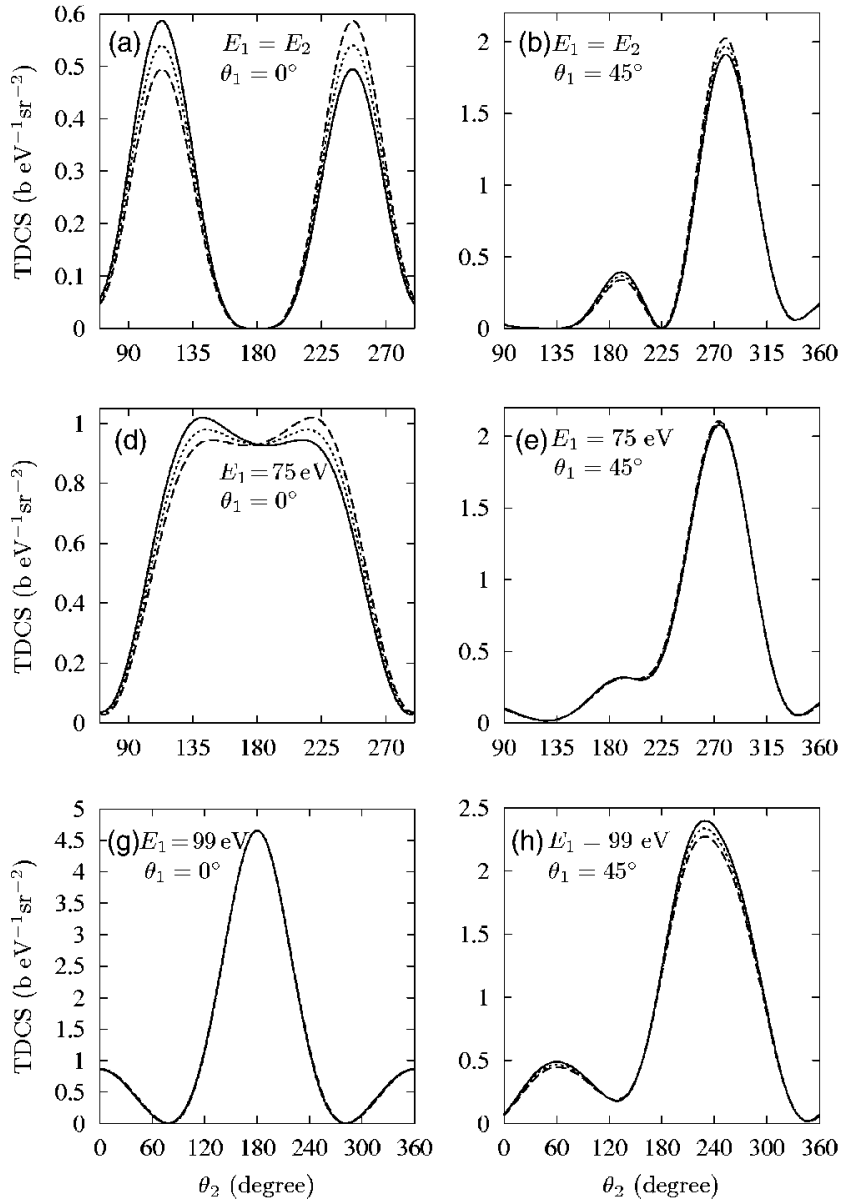

For equal energy sharing, Fig. 6 shows that the nondipole asymmetry is largest for $\theta_{1}=0^{\circ}$. For the intermediate regime of energy sharing, i.e., $E_{1}=75 \mathrm{eV}$ and $E_{2}=25 \mathrm{eV}$, the asymmetry is largest for $\theta_{1}=0^{\circ}$ and $\theta_{1}=90^{\circ}$. For the limiting case of extremely asymmetric energy sharing, $E_{1}=99 \mathrm{eV}$ and $E_{2}$ $=1 \mathrm{eV}$, we find the largest nondipole asymmetry at $\theta_{1}=90^{\circ}$. The nondipole modifications of the angular distributions for particular values of $\theta_{1}$ are similar to those predicted for the excess energy of $20 \mathrm{eV}$, i.e., the TDCS is shifted in the forward direction, etc.

Our TDCS predictions at this excess energy for DPI by circularly polarized light are shown in Fig. 7. The geometrical arrangement is shown in Fig. 2(b), i.e., the electron having energy $E_{1}$ is always detected along the $x$ axis. (This geometry is identical to that employed in Fig. 5.) For the case of equal energy sharing, the nondipole asymmetries are large for detection of the second electron in either the $x z$ plane or the $y z$ plane. For the case $E_{1}=75 \mathrm{eV}$, the asymmetry is largest for detection of the second electron in the $x z$ plane. For extremely asymmetric energy sharing, shown in Figs. 7(c) and 7(f), the asymmetry is vanishingly small.

\section{Excess energy of $450 \mathrm{eV}$}

The excess energy of $450 \mathrm{eV}$ is the highest one for which there exist experimental measurements of the TDCS [12]. The analysis of DPI cross sections at this excess energy is of great interest as GS electron correlations (which include the
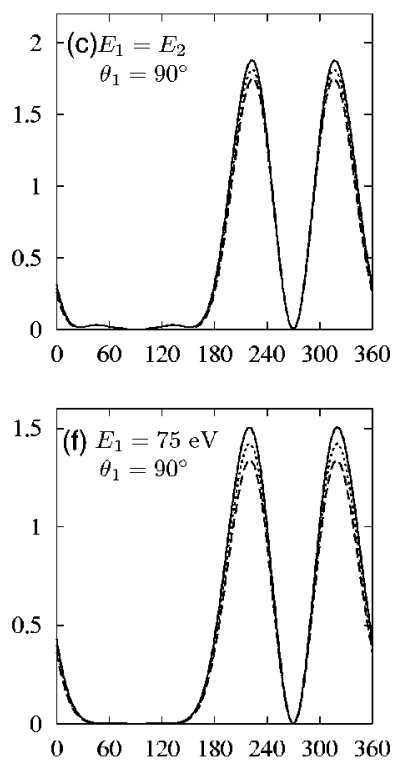

FIG. 6. Present results for the DPI TDCS at an excess energy of $100 \mathrm{eV}$ for linear polarization and coplanar geometry for three energy sharings and ejection angles. Full curves: dipole-quadrupole results; dashed curves: dipolequadrupole results for the inverted direction of the photon beam; dotted curves: the EDA results. (Note that $1.0 \mathrm{~b}=1.0 \times 10^{-24} \mathrm{~cm}^{2}$.)

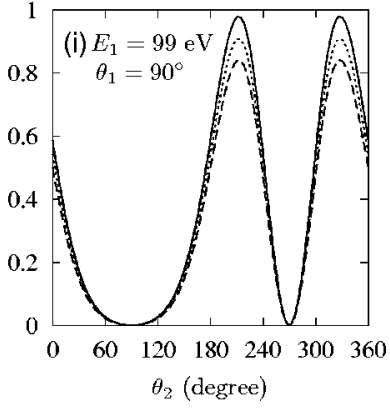

shake-off process) begin to play an important role in DPI and to interfere with FS correlations (which include the knockout process) [12].

In Fig. 8, we present our predictions for the DPI TDCS for the same two energy sharings and for the same electron ejection angle, $\theta_{1}=0^{\circ}$, for which experimental measurements [12] and our previous EDA calculations [16] have been carried out. Our present results include both FS and GS correlations in both the electric-dipole and electric-quadrupole transition amplitudes. A single basis set of $Z=2$ Coulomb functions is used; therefore, the results shown are gaugeinvariant. Despite the discrepancies of our predictions with the experimental data at relatively small mutual ejection angles $0^{\circ} \lesssim \theta_{12} \lesssim 90^{\circ}$ [see, e.g., Fig. 8(b)], for which a nonperturbative treatment is necessary, our account of the lowest-order nondipole correction allows us to provide further insight into the experimental results [12].

In both our theoretical calculations and the experimental measurements [12], the coplanar geometry is used, as shown in Fig. 8(a). Our calculations assume that the photon wave vector $\mathbf{k}$ is directed downward; the experimental measurements [12], which did not search for nondipole effects, do not specify whether the vector $\mathbf{k}$ is directed upward or downward [40]. Our full curves show the TDCS with account of dipole-quadrupole terms, while our dashed curves show our prior EDA results [16].

As seen in Fig. 8, when the slower electron is ejected along the photon polarization vector $\hat{\boldsymbol{\epsilon}}$, our dipole- 

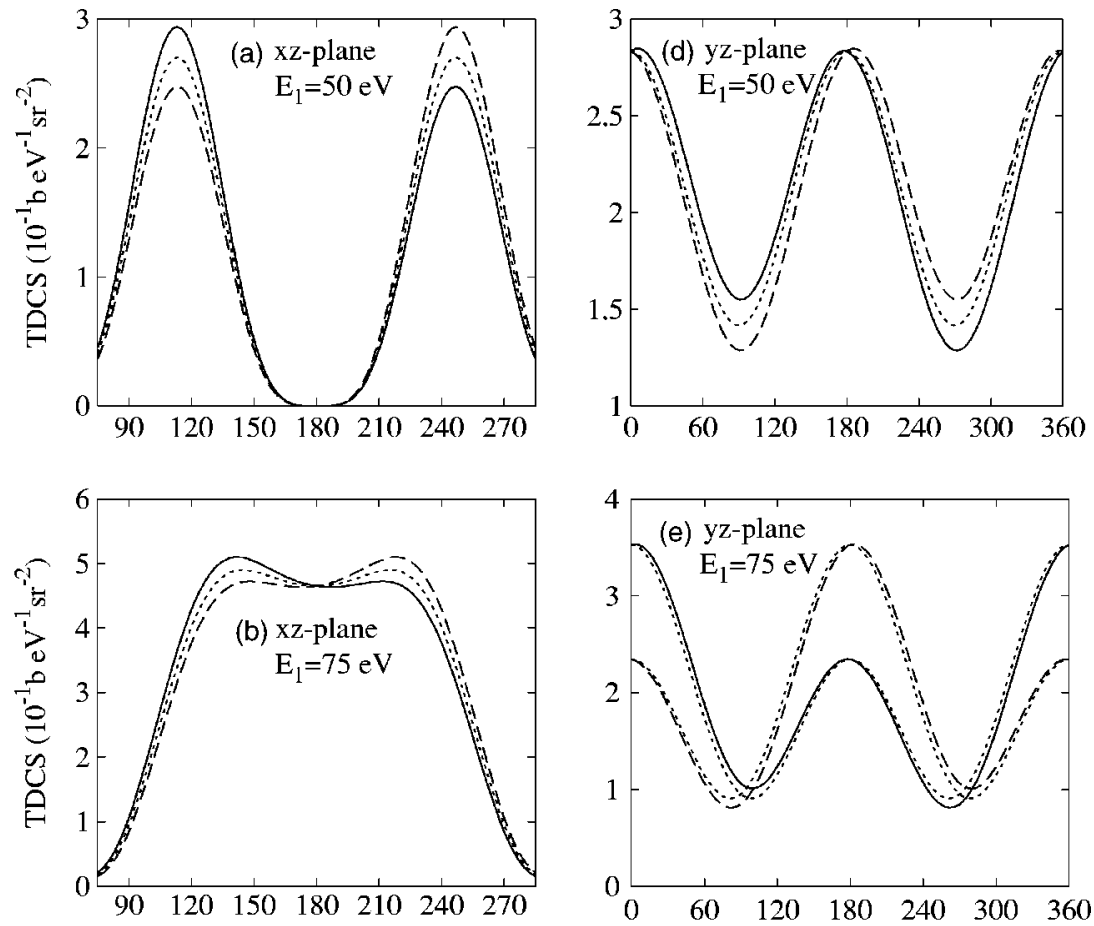

FIG. 7. Present TDCS results at an excess energy of $100 \mathrm{eV}$ for circular polarization $(\xi=+1)$. Energy sharings and ejection angles are as indicated. Full curves: dipole-quadrupole results; dashed curves: dipole-quadrupole results for the inverted photon beam direction; dotted curves: EDA results. (Note that $1.0 \mathrm{~b}=1.0$ $\times 10^{-24} \mathrm{~cm}^{2}$.)
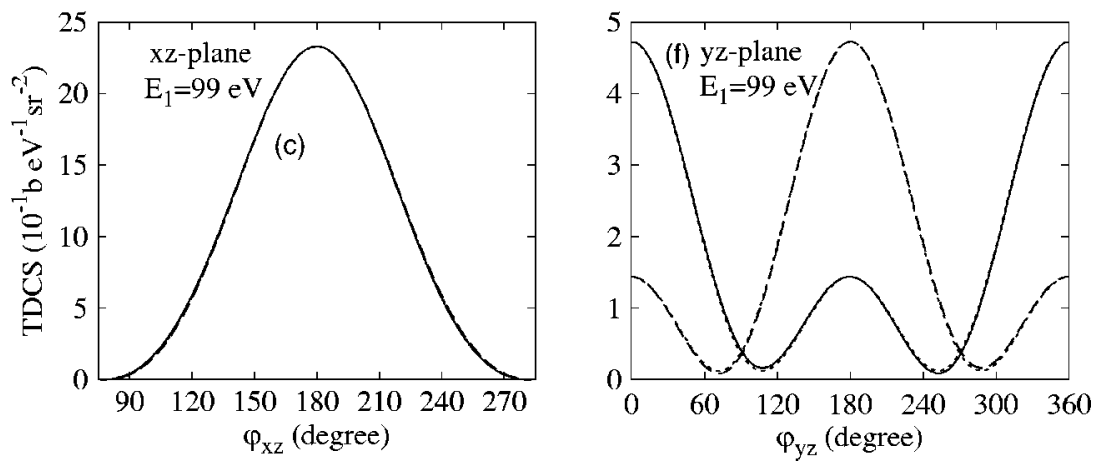

quadrupole predictions for the angular distribution of the faster electron exhibit a noticeable forward-backward asymmetry, i.e., they are shifted along the direction of the vector $\mathbf{k}$, as expected intuitively. A similar asymmetry may be noticed also in the experimental data of Ref. [12] shown in Fig. 8. This is in contrast to the EDA predictions given in Refs. $[12,16,41]$. When the faster electron is ejected along the photon polarization direction, our numerical results show that the nondipole shift of the angular distribution of the slower electron is rather small; consequently, results for this case are not shown.

The form of the quadrupole radial matrix elements [shown in Eqs. (A1) and (A10) in Appendix A] is very similar to that of the dipole radial matrix elements in Eqs. (A2) and (A17) in Ref. [16]. Therefore, despite the considerable discrepancy seen in Fig. 8(b) between our EDA prediction for the TDCS and the experimental data, we expect that the relative magnitudes of the nondipole asymmetries should not be affected significantly by the choice of theoretical model used to account for electron correlations. Indeed, we find reasonable agreement between the experimental data [12] and our LOPT predictions in Fig. 8(b) for the ratio of the difference of the TDCSs in the forward and backward half- planes to the TDCS in the forward half-plane, i.e.,

$$
R=\frac{\sigma\left(2 \pi-\theta_{2}\right)-\sigma\left(\theta_{2}\right)}{\sigma\left(2 \pi-\theta_{2}\right)},
$$

for several values of the angle $\theta_{2}$ (see Table I). Given that the asymmetries in our results and those seen in the experimental data (cf. Fig. 8 and Table I) are in qualitative agreement (for our choice of the vector $\mathbf{k}$ direction in Fig. 8) and are of comparable magnitude, we believe that it is reasonable to consider the experimental results in Ref. [12] a first-time observation of the signatures of nondipole effects in the TDCS for the DPI process, although future experiments and more elaborate calculations would be desirable to confirm this hypothesis.

\section{B. Circular dichroism at equal energy sharing}

In this subsection, we conclude our analysis of nondipole effects in DPI of He by presenting results for one of its most interesting manifestations: the nonzero $\mathrm{CD}$ effect at equal energy sharing. As discussed in Sec. II C 2, this effect originates from the nonzero imaginary part of the coefficient $c_{3}$ in Eq. (32). Our results for the equal-energy-sharing TDCSs for 

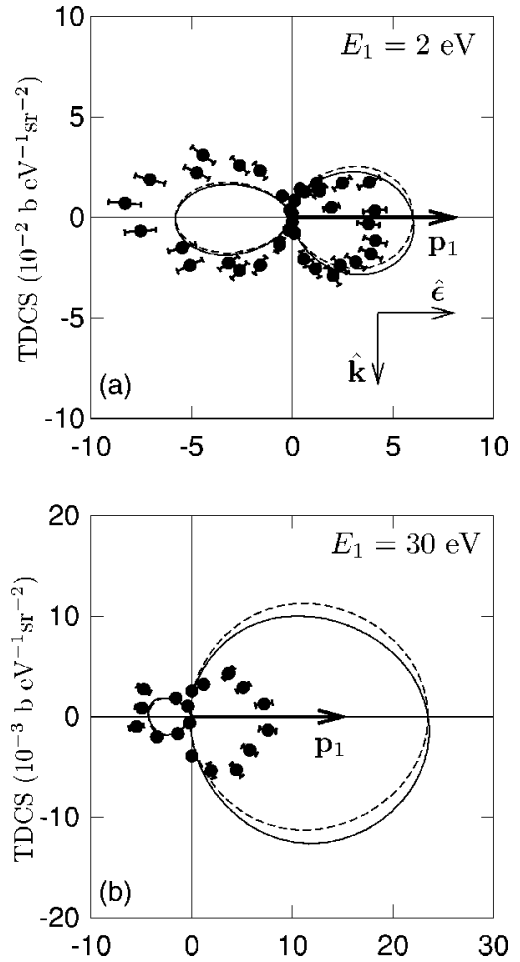

FIG. 8. Comparison of the present TDCS results for an excess energy of $450 \mathrm{eV}$ for linear polarization and for coplanar geometry with the normalized experimental data of Ref. [12]. The direction of the photon wave vector $\hat{\mathbf{k}}$ and polarization $\hat{\boldsymbol{\epsilon}}$ is as shown in (a); the electron having momentum $p_{1}$ (and energy $E_{1}$ ) is ejected along $\epsilon$. Full curves: dipole-quadrupole results; dashed curves: EDA results. Note that both the experimental data and our nondipole results exhibit a noticeable forward-backward asymmetry as compared to our EDA results: the angular distributions of the fast electron are shifted along the direction of the vector $\mathbf{k}$, especially in the angular ranges $0^{\circ}<\theta_{2}<90^{\circ}$ and $270^{\circ}<\theta_{2}<360^{\circ}$; cf. Table I. (Note that $1.0 \mathrm{~b}$ $=1.0 \times 10^{-24} \mathrm{~cm}^{2}$.)

DPI by circularly polarized photons with $\xi=+1$ and $\xi=-1$ are presented in Fig. 9. The geometry is as shown in Fig. 2(a), i.e., both electrons are detected in the plane which makes an angle of $45^{\circ}$ with both the light propagation direction, $\hat{\mathbf{k}}$, and with the polarization plane. The TDCSs at the
TABLE I. Experimental and theoretical ratio of the difference of the TDCSs for $E_{1}=30 \mathrm{eV}, E_{2}=420 \mathrm{eV}$ in the forward and backward half-planes to the TDCS in the forward half-plane [cf. Fig. 8(b)] as defined in Eq. (69) for four ejection angles $\theta_{2} . R^{\exp }$, experimental value (calculated using data from Ref. [12]; $R^{\text {th }}$, theoretical value (LOPT).

\begin{tabular}{ccc}
\hline \hline $\begin{array}{c}\theta_{2} \\
(\text { radian })\end{array}$ & $R^{\exp }$ & $R^{\text {th }}$ \\
\hline 0.174 & 0.049 & 0.051 \\
0.516 & 0.117 & 0.143 \\
0.868 & 0.181 & 0.212 \\
1.216 & 0.398 & 0.229 \\
\hline \hline
\end{tabular}

excess energies of 20 and $100 \mathrm{eV}$ were calculated with account of FS correlations only, while at $450 \mathrm{eV}$ both FS and GS correlations were included.

Figure 9(a) shows that at an excess energy of $20 \mathrm{eV}$, the effect is so small that no difference is observed between the TDCSs for $\xi=+1$ and $\xi=-1$, as expected within the EDA. For the excess energies of $100 \mathrm{eV}$ and especially $450 \mathrm{eV}$, however, there are clear discrepancies between the TDCSs calculated for $\xi=+1$ and $\xi=-1$, thus indicating the nonzero equal-energy-sharing CD effect. One sees, however, that in Fig. 9(c) the TDCS curves exhibit an unusual behavior in the vicinity of $\theta_{12}=180^{\circ}$ that is different from Figs. 9(a) and 9(b). This difference in the behavior is due to the fact that in Figs. 9(a) and 9(b) only FS correlations are taken into account, while in Fig. 9(c) both FS and GS correlations are included.

Treatment of electron correlations for the equal-energysharing regime in Fig. 9(c) is particularly difficult by means of approximate methods, such as the LOPT employed here. On one hand, since the photoelectron energies are high, the final-state wave function exhibits rapid oscillations and GS correlations must generally be included, despite the fact that one of the GSC amplitude components, describing shake-off, should not give a substantial contribution. On the other hand, the excess energy is shared equally between photoelectrons and, as has been shown previously for the case of low photon energies $[14,16]$, this is realized primarily via the knock-out mechanism. The regime of symmetric energy sharing for
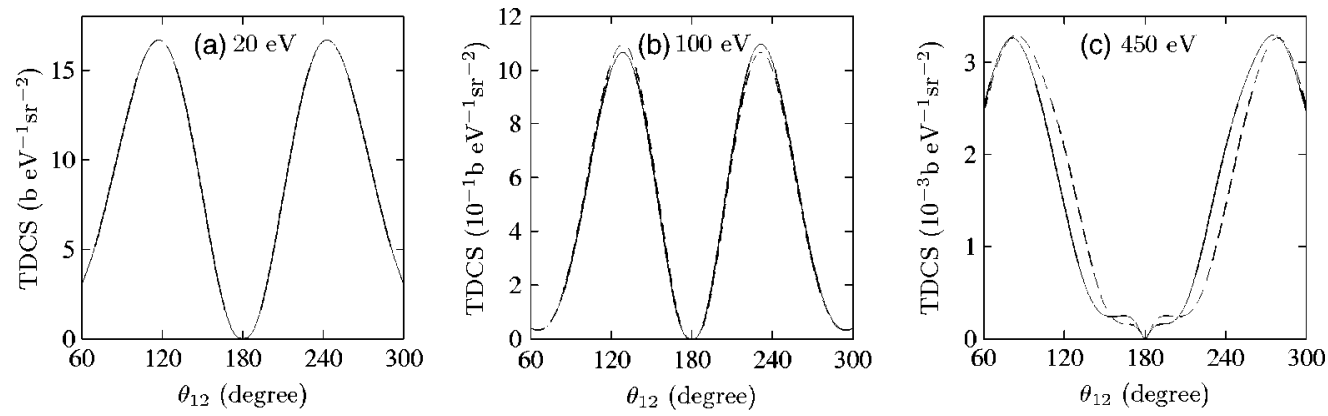

FIG. 9. Present results exhibiting the equal-energy-sharing CD effect, i.e., the sensitivity of the dipole-quadrupole equal-energy-sharing TDCS to the sign of the degree of circular polarization, $\xi$, for three excess energies. The geometry is as shown in Fig. 2(a). Full curves: $\xi=+1$; dashed curves: $\xi=-1$. (Note that $1.0 \mathrm{~b}=1.0 \times 10^{-24} \mathrm{~cm}^{2}$.) 
high excess energy has not been analyzed in detail, either by us or by others. We therefore do not expect Fig. 9(c) to provide more than qualitative agreement with future experiments. We note also that upon neglecting the GSC diagram in Fig. 9(c), the result for the TDCSs for an excess energy of $450 \mathrm{eV}$ becomes very similar to that in Fig. 9(b), with the relative magnitude of the difference between the full and dashed curves at the TDCS maxima of the order of $5 \%$.

\section{SUMMARY}

In the present work, we have performed a systematic analysis of lowest-order retardation effects in the fully differential cross section for DPI of He. We have presented model-independent parametrizations for the DPI quadrupole transition amplitude and for the dipole-quadrupole TDCSs for linear, circular, and elliptic polarizations. These parametrizations may be useful for analyzing data in future experiments.

In our theoretical analyses, we have considered three representative values of the excess energies, 20, 100, and 450 $\mathrm{eV}$. We have found that observation of lowest-order nondipole effects in the TDCS is feasible for a number of geometrical arrangements depending on the photon polarization and the excess energy sharing. Our predictions for the TDCS forward-backward asymmetries indicate that they may be observed at excess energies as low as $20 \mathrm{eV}$. Our predictions for the TDCS at the excess energy of $450 \mathrm{eV}$ reveal a noticeable forward-backward asymmetry which is in qualitative agreement with existing experimental measurements at this excess energy. Finally, we have presented our predictions for the equal-energy-sharing $\mathrm{CD}$ effect at the same three excess energies. Although small, this effect may still be observable in the near future, as experimental techniques are being continuously improved. The present work, together with Refs. $[17-19,33,34]$, thus clarifies another aspect of the fundamental process of complete fragmentation of an interacting threebody Coulomb system by one photon, i.e., the effect of the spatial inhomogeneity of the light wave on this breakup process.

The manifestations of nondipole effects in the cross sections integrated over the ejection angles of one or both electrons (double or single differential cross sections, respectively) are certainly of fundamental and practical interest. We note here that it is possible to derive the simple $a b$ initio parametrizations for these cross sections in terms of the reduced two-electron matrix elements of the operators $\mathbf{D}$ and $Q_{2}$. These parametrizations together with numerical LOPT results will be presented elsewhere.

\section{ACKNOWLEDGMENTS}

This work was supported in part by the U.S. Department of Energy, Office of Science, Division of Chemical Sciences, Geosciences, and Biosciences, under Grant No. DE-FG0396ER14646, by RFBR Grant No. 04-02-16350, and by the joint Grant No. VZ-010-0 of the CRDF and the RF Ministry of Education (N.L.M.). This work was completed utilizing the Research Computing Facility of the University of Nebraska-Lincoln.

\section{APPENDIX A: QUADRUPOLE RADIAL MATRIX ELEMENTS}

\section{Evaluation of the FSC quadrupole matrix elements}

Quadrupole radial matrix elements describing FSC have the form

$$
\begin{aligned}
\mathcal{Q}_{F}^{\left(l_{1}, l_{2}\right)}\left(p_{1}, p_{2}\right)= & \int_{0}^{\infty} d r r^{2} R_{p_{1} l_{1}}(r) u_{p_{2} l_{2}}(r) \\
& \times \int_{0}^{\infty} d r^{\prime} r^{\prime 3} g_{2}\left(\mathcal{E} ; r, r^{\prime}\right)\left(d / d r^{\prime}\right) R_{1 s}\left(r^{\prime}\right),
\end{aligned}
$$

where $R_{1 s}(r)$ is a hydrogenic ground-state orbital corresponding to the effective (screened) charge $Z_{s}$,

$$
R_{1 s}(r)=2 \sqrt{Z_{s}^{3}} e^{-Z_{s} r} .
$$

The function $u_{p_{2} l_{2}}(r)$ in Eq. (A1) is defined by the following integral:

$$
u_{p_{2} l_{2}}(r)=\int_{0}^{\infty} d r^{\prime \prime} r^{\prime \prime 2} R_{p_{2} l_{2}}\left(r^{\prime \prime}\right) \frac{r_{<}^{l_{2}}}{r_{>}^{l_{2}+1}} R_{1 s}\left(r^{\prime \prime}\right),
$$

which was evaluated in Ref. [16],

$$
u_{p l}(r)=\mathcal{C}_{p l} \int_{0}^{1} d x x^{l+i \eta}(1-x)^{l-i \eta} \chi_{p l}(r, x),
$$

where $\mathcal{C}_{p l}$ and $\chi_{p l}(r, x)$ are given by

$$
\begin{gathered}
\mathcal{C}_{p l}=\frac{(2 p)^{l+1} e^{\pi \eta / 2}}{|\Gamma(l+1+i \eta)|}, \\
\chi_{p l}(r, x)=\int_{0}^{\infty} d r^{\prime \prime} r^{\prime \prime l+2} e^{-\tau r^{\prime \prime}} \frac{r_{<}^{l}}{r_{>}^{l+1}} \\
=\frac{(2 l+2) !}{\tau\left(\tau^{2} r\right)^{l+1}}\left\{1-e^{-\tau r} \sum_{k=0}^{2 l+1}\left(1-\frac{\delta_{k, 2 l+1}}{2 l+2}\right) \frac{(\tau r)^{k}}{k !}\right\},
\end{gathered}
$$

and where $\tau=Z_{s}+i p(1-2 x), \quad r_{<}=\min \left(r, r^{\prime \prime}\right), \quad$ and $\quad r_{>}$ $=\max \left(r, r^{\prime \prime}\right)$. With the definitions given above, the integrals over $r$ and $r^{\prime}$ in Eq. (A1) are evaluated in the same way as are similar integrals for the dipole radial matrix elements in Ref. [16]. The expression for $\mathcal{Q}_{F}^{\left(l_{1}, l_{2}\right)}$ has the following form: 


$$
\begin{aligned}
\mathcal{Q}_{F}^{\left(l_{1}, l_{2}\right)}= & \frac{2^{8} \nu Z_{s}^{4}\left(2 p_{1}\right)^{l_{1}}}{\left(1+Z_{s} \nu\right)^{6}} \frac{\left(2 l_{2}+2\right) !}{\left(2 l_{1}+1\right) !} \times \mathcal{C}_{p_{1} l_{1}} \mathcal{C}_{p_{2} l_{2}} \int_{0}^{1} d u \frac{u^{2-Z \nu}}{(1+\gamma u)^{6}} \\
& \times \int_{0}^{1} d x \frac{x^{l_{2}+i \eta_{2}}(1-x)^{l_{2}-i \eta_{2}}}{\tau^{2 l_{2}+3}}\left\{J_{l_{1}-l_{2}+4,2 l_{1}+2}^{l_{1}+1+i \eta_{1}, 2 i p_{1}}(\lambda)\right. \\
& \left.-\sum_{k=0}^{2 l_{2}+1}\left(1-\frac{\delta_{k, 2 l_{2}+1}}{2 l_{2}+2}\right) \frac{\tau^{k}}{k !} J_{l_{1}-l_{2}+1+i+4,2 l_{1}+2}^{l_{1}+1+i \eta_{1}, 2 i p_{1}}(\lambda+\tau)\right\},
\end{aligned}
$$

where

$$
\begin{gathered}
\lambda=i p_{1}+\frac{1}{\nu} \frac{1-\gamma u}{1+\gamma u}, \\
\gamma=\left(1-Z_{s} \nu\right) /\left(1+Z_{s} \nu\right),
\end{gathered}
$$

and where $\nu \equiv \nu_{F}=1 / \sqrt{-2\left(E_{p_{1}}+E_{p_{2}}-\epsilon_{1 s}\right)}=i\left|\nu_{F}\right|$. The function $J_{k, m}^{\kappa, \beta}(\sigma)$ in Eq. (A7) is

$J_{k m}^{\kappa \beta}(\sigma)=\int_{0}^{\infty} d t t^{k-1} e^{-\sigma t} \Phi(\kappa ; m ; \beta t)=\frac{(k-1) !}{\sigma^{k}}{ }_{2} F_{1}\left(\kappa, k ; m ; \frac{\beta}{\sigma}\right)$,

where the hypergeometric function ${ }_{2} F_{1}$ reduces to elementary functions for positive integer values of $k$ and $m$ (as in our case).

\section{Evaluation of the GSC quadrupole matrix elements}

The radial matrix elements entering the GSC quadrupole transition amplitude are given by

$$
\mathcal{Q}_{G}^{\left(l_{1}, l_{2}\right)}\left(p_{1}, p_{2}\right)=\int_{0}^{\infty} d r r^{3} R_{p_{1} l_{1}}(r) \hat{Q}\left(l_{1}, l_{2}\right) I_{p_{2} l_{2}}(r),
$$

where $\hat{Q}\left(l_{1}, l_{2}\right)$ is the differential operator,

$$
\hat{Q}\left(l_{1}, l_{2}\right)=\frac{d}{d r}+\frac{\delta_{l_{1} l_{2}}(3 / 2)+\delta_{l_{1} l_{2}-2}\left(l_{2}+1\right)-\delta_{l_{1} l_{2}+2} l_{2}}{r} .
$$

The function $I_{p_{2} l_{2}}(r)$ is defined as follows [16]:

$$
\begin{aligned}
I_{p_{2} l_{2}}(r)= & \int_{0}^{\infty} d r^{\prime} r^{\prime 2} g_{l_{2}}\left(E ; r, r^{\prime}\right) u_{p_{2} l_{2}}\left(r^{\prime}\right) R_{1 s}\left(r^{\prime}\right) \\
= & 2^{2 l_{2}+5} \nu\left(l_{2}+1\right) Z_{s}^{3} \mathcal{C}_{p_{2} l_{2}} \\
& \times \int_{0}^{1} d u u^{l_{2}-Z \nu} \int_{0}^{1} d x \frac{x^{l_{2}+i \eta_{2}}(1-x)^{l_{2}-i \eta_{2}}}{\tau^{2 l_{2}+3}} \mathcal{F}_{p_{2} l_{2}}(r), \\
\mathcal{F}_{p_{2} l_{2}}(r)= & r^{l^{2}}\left[e^{-\lambda_{1}^{\prime} r} \frac{\Phi\left(2 l_{2} ; 2 l_{2}+2 ; t_{1} r\right)}{\alpha^{2}[\nu(1-u)]^{2 l_{2}}}\right. \\
& \left.-e^{-\lambda_{2}^{\prime} r} \sum_{k=-1}^{2 l_{2}} b_{k}^{\left(l_{2}\right)} \frac{\Phi\left(k ; 2 l_{2}+2 ; t_{2} r\right)}{[\nu(1-u)]^{k}}\right], \quad \text { (A } 1
\end{aligned}
$$

where

$$
\begin{gathered}
\alpha=\left(1+Z_{s} \nu\right)(1+\gamma u), \quad \xi=\frac{\nu}{1+Z_{s} \nu}, \\
\beta_{1}=\frac{4 u}{\left(1+Z_{s} \nu\right)(1+\gamma u)},
\end{gathered}
$$

$$
\begin{gathered}
\beta_{2}=\frac{4 u}{\left(1+Z_{s} \nu\right)[1+\gamma u+\xi \tau(1-u)]}, \\
\lambda_{1}^{\prime}=\frac{1}{\nu} \frac{1-\gamma u}{1+\gamma u}, \quad \lambda_{2}^{\prime}=\frac{1}{\nu} \frac{1-\gamma u+\xi \tau(1+u)}{1+\gamma u+\xi \tau(1-u)}, \\
t_{1}=-\frac{1}{\nu} \frac{\beta_{1}}{1-u}, \quad t_{2}=-\frac{1}{\nu} \frac{\beta_{2}}{1-u}, \\
b_{k}^{\left(l_{2}\right)}=\left(1-\frac{\delta_{k,-1}}{2 l_{2}+2}\right) \frac{\left(2 l_{2}+1-k\right) \tau^{2 l_{2}-k}}{[\alpha+\nu \tau(1-u)]^{2 l_{2}+2-k} .}
\end{gathered}
$$

The function $\Phi(a ; c ; x) \equiv{ }_{1} F_{1}(a ; c ; x)$ is a confluent hypergeometric function having one upper parameter, $a$, and one lower parameter, $c$ [42]. The parameters $\gamma$ and $\nu$ are $\gamma=(1$ $\left.-Z_{s} \nu\right) /\left(1+Z_{s} \nu\right)$ and $\nu \equiv \nu_{G}=1 / \sqrt{-2\left(2 \epsilon_{1 s}-E_{p_{2}}\right)}$. Note that because the parameter $\nu$ here is a positive real number, the radial matrix elements $\mathcal{Q}_{G}^{\left(l_{1} l_{2}\right)}\left(p_{1}, p_{2}\right)$ are real despite the appearance of complex quantities in their definition.

With the above definitions, the integrals over $r$ in Eq. (A10) may be reduced to the following integral of two confluent hypergeometric functions having the same value of the (integer) lower parameter $m$ :

$$
\begin{aligned}
& \int_{0}^{\infty} d r e^{-\sigma r} r^{m-1+n} \Phi\left(\kappa ; m ; 2 i p_{1} r\right) \Phi\left(k ; m ; \frac{-\beta r}{\nu(1-u)}\right) \\
& \quad=[\nu(1-u)]^{k} K_{k \beta}^{\kappa m n}(\sigma) .
\end{aligned}
$$

For the simplest case of $n=0$, one has $K_{k \beta}^{k m 0}(\sigma) \equiv \mathcal{L}_{k \beta}^{\kappa m}(\sigma)$, where

$$
\begin{aligned}
\mathcal{L}_{k \beta}^{\kappa m}(\sigma) \equiv & \frac{(m-1) ! \sigma^{k-m}}{[\sigma \nu(1-u)+\beta]^{k}}\left(\frac{\sigma}{\sigma-2 i p_{1}}\right)^{\kappa} \\
& \times{ }_{2} F_{1}(\kappa, k ; m ; z), \\
z= & \frac{-2 i p_{1} \beta}{\left(\sigma-2 i p_{1}\right)[\sigma \nu(1-u)+\beta]} .
\end{aligned}
$$

For integer $k$ and $m$, the hypergeometric function ${ }_{2} F_{1}$ in the above equation reduces to elementary functions. The result for $K_{k \beta}^{k m n}(\sigma)$ at integer $n>0$ can be obtained by differentiation, 


$$
K_{k \beta}^{\kappa m n}(\sigma)=(-1)^{n} \frac{d^{n}}{d \sigma^{n}} \mathcal{L}_{k \beta}^{\kappa m}(\sigma)
$$

In order to apply the formulas (A14) and (A15), it is necessary to transform the confluent hypergeometric functions $\Phi(a ; c ; x)$ in Eq. (A10) [cf. Eqs. (49) and (A11)] so that they have the same value of the lower parameter $c$, while keeping the power of $r$ in the integrand of Eq. (A10) equal to $(c-1)$. The action of the differential operator $\hat{Q}\left(l_{1}, l_{2}\right)$ in Eq. (A10) can be shifted from $I_{p_{2} l_{2}}(r)$ to $R_{p_{1} l_{1}}(r)$ by an integration by parts. Because differentiation of the function $\Phi$ increases the values of both of its parameters by 1 , it is convenient to have $\hat{Q}\left(l_{1}, l_{2}\right)$ act on the function $\Phi(a ; c ; x)$ that has the lower value of the parameter $c$. In other words, for angular momenta $\left(l_{1}=l+2, l_{2}=l\right)$ it is convenient to use Eq. (A10) directly, while for angular momenta $\left(l_{1}=l, l_{2}=l+2\right)$ it is more convenient to shift the action of $\hat{Q}\left(l_{1}, l_{2}\right)$ to $R_{p_{1} l_{1}}(r)$. For this latter case, Eq. (A10) is replaced by

$$
\mathcal{Q}_{G}^{\left(l_{1}, l_{2}\right)}\left(p_{1}, p_{2}\right)=\int_{0}^{\infty} d r r^{3} I_{p_{2} l_{2}}(r) \hat{Q}^{\prime}\left(l_{1}, l_{2}\right) R_{p_{1} l_{1}}(r),
$$

where

$$
\hat{Q}^{\prime}\left(l_{1}, l_{2}\right)=-\frac{\partial}{\partial r}+\frac{\delta_{l_{1} l_{2}-2} l_{1}-\delta_{l_{1} l_{2}+2}\left(l_{1}+1\right)-\delta_{l_{1} l_{2}}(3 / 2)}{r} .
$$

The case of $l_{1}=l_{2}=l$ can be treated either way.

$$
\text { a. Case } l_{1}=l+2, l_{2}=l
$$

For this case, we obtain

$$
\begin{aligned}
\hat{Q}(l+ & 2, l) \mathcal{F}_{p_{2} l}(r) \\
= & \frac{r^{l} e^{-\lambda_{1}^{\prime} r}}{\alpha^{2}[\nu(1-u)]^{2 l}}\left[t_{1} \frac{c_{0}}{c_{2}} \Phi\left(c_{1} ; c_{3} ; t_{1} r\right)-\lambda_{1}^{\prime} \Phi\left(c_{0} ; c_{2} ; t_{1} r\right)\right] \\
& -r^{l} e^{-\lambda_{2}^{\prime} r} \sum_{k=-1}^{2 l} b_{k}^{(l)} \frac{t_{2} \frac{k}{c_{2}} \Phi\left(k+1 ; c_{3} ; t_{2} r\right)-\lambda_{2}^{\prime} \Phi\left(k ; c_{2} ; t_{2} r\right)}{[\nu(1-u)]^{k}},
\end{aligned}
$$

where we have introduced the notations $a_{k} \equiv i \eta_{1}+l+k$ and $\quad c_{k} \equiv 2 l+k$. The function $R_{p_{1} l+2}(r)$ $=A_{p_{1} l+2} r^{l+2} e^{-i p_{1} r} \Phi\left(a_{3} ; c_{6} ; 2 i p_{1} r\right) \quad\left[\right.$ where $\quad A_{p_{1} l} \equiv\left[\left(2 p_{1}\right)^{l} /(2 l\right.$ $\left.+1) !] C_{p_{1} l}\right]$ may be rewritten in terms of functions $\Phi$ having their lower parameters equal to either $c_{3}$ or $c_{2}$ by using the formulas

$$
\begin{gathered}
\Phi(c+3)=\frac{[c]_{2}}{x^{3}}[\Phi-3 \Phi(a-1)+3 \Phi(a-2)-\Phi(a-3)], \\
\Phi(c+4)=\frac{[c]_{3}}{x^{4}}[\Phi-4 \Phi(a-1)+6 \Phi(a-2) \\
-4 \Phi(a-3)+\Phi(a-4)],
\end{gathered}
$$

where we have used the abbreviated notations $\Phi$ $\equiv \Phi(a ; c ; x), \quad \Phi(a \pm n) \equiv \Phi(a \pm n ; c ; x), \quad$ and $\quad \Phi(c \pm n)$ $\equiv \Phi(a ; c \pm n ; x)$; also $[a]_{n} \equiv a(a+1) \ldots(a+n)$. Equations (A18) are obtained using the known relation [42]

$$
x \Phi(c+1)=c \Phi-c \Phi(a-1) .
$$

Using Eq. (A13) with $n=0$, one obtains the final result

$$
\begin{aligned}
\mathcal{Q}_{G}^{(l+2, l)}\left(p_{1}, p_{2}\right)= & 2^{2 l+5} \nu(l+1) Z_{s}^{3} A_{p_{1} l+2} \mathcal{C}_{p_{2}} \int_{0}^{1} d u u^{l-Z v} \int_{0}^{1} d x \frac{x^{l+i \eta_{2}}(1-x)^{l-i \eta_{2}}}{\tau^{2 l+3}} \frac{1}{\left(2 i p_{1}\right)^{3}}\left\{\gamma _ { 1 } \left[\mathcal{L}_{c_{1}, \beta_{1}}^{a_{3}, c_{3}}\left(\lambda_{1}\right)-3 \mathcal{L}_{c_{1}, \beta_{1}}^{a_{2}, c_{3}}\left(\lambda_{1}\right)+3 \mathcal{L}_{c_{1}, \beta_{1}}^{a_{1}, c_{3}}\left(\lambda_{1}\right)\right.\right. \\
& \left.-\mathcal{L}_{c_{1}, \beta_{1}}^{a_{0}, c_{3}}\left(\lambda_{1}\right)\right]-\gamma_{2}\left[\mathcal{L}_{c_{0}, \beta_{1}}^{a_{3}, c_{2}}\left(\lambda_{1}\right)-4 \mathcal{L}_{c_{0}, \beta_{1}}^{a_{2}, c_{2}}\left(\lambda_{1}\right)+6 \mathcal{L}_{c_{0}, \beta_{1}}^{a_{1}, c_{2}}\left(\lambda_{1}\right)-4 \mathcal{L}_{c_{0}, \beta_{1}}^{a_{0}, c_{2}}\left(\lambda_{1}\right)+\mathcal{L}_{c_{0}, \beta_{1}}^{a_{-1}, c_{2}}\left(\lambda_{1}\right)\right] \\
& -\sum_{k=-1}^{2 l} b_{k}^{(l)}\left\{\gamma_{3} k\left[\mathcal{L}_{k+1, \beta_{2}}^{a_{3}, c_{3}}\left(\lambda_{2}\right)-3 \mathcal{L}_{k+1, \beta_{2}}^{a_{2}, c_{3}}\left(\lambda_{2}\right)+3 \mathcal{L}_{k+1, \beta_{2}}^{a_{1}, c_{3}}\left(\lambda_{2}\right)-\mathcal{L}_{k+1, \beta_{2}}^{a_{0}, c_{3}}\left(\lambda_{2}\right)\right]-\gamma_{2}\left[\mathcal{L}_{k, \beta_{2}}^{a_{3}, c_{2}}\left(\lambda_{2}\right)-4 \mathcal{L}_{k, \beta_{2}}^{a_{2}, c_{2}}\left(\lambda_{2}\right)\right.\right. \\
& \left.\left.+6_{k, \beta_{2}}^{a_{1}, c_{2}}\left(\lambda_{2}\right)-4 \mathcal{L}_{k, \beta_{2}}^{a_{0}, c_{2}}\left(\lambda_{2}\right)+\mathcal{L}_{k, \beta_{2}}^{a_{-1}, c_{2}}\left(\lambda_{2}\right)\right]\right\}
\end{aligned}
$$

where

$$
\gamma_{1}=-\left[c_{3}\right]_{2} \frac{c_{0}}{c_{2}} \frac{\beta_{1}}{\alpha^{2}}, \quad \gamma_{2}=\frac{\left[c_{2}\right]_{3}}{2 i p_{1}} \frac{\lambda_{1}^{\prime}}{\alpha^{2}}, \quad \gamma_{3}=-\frac{\left[c_{3}\right]_{2}}{c_{2}} \beta_{2}, \quad \gamma_{4}=\frac{\left[c_{2}\right]_{3}}{2 i p_{1}} \lambda_{2}^{\prime}, \quad \lambda_{1}=\lambda_{1}^{\prime}+i p_{1}, \quad \lambda_{2}=\lambda_{2}^{\prime}+i p_{1} .
$$




\section{b. Case $l_{1}=l_{2}=l$}

Using Eq. (A19), one obtains

$$
\begin{aligned}
\hat{Q}^{\prime}(l, l) R_{p_{1} l}(r)= & \left(i \eta_{1}-1 / 2\right) R_{p_{1} l}(r) / r+i p_{1} R_{p_{1} l}(r) \\
& -a_{1} A_{p_{1}} l^{l-1} e^{-i p_{1} r} \Phi\left(a_{2} ; c_{2} ; 2 i p_{1} r\right) .
\end{aligned}
$$

The integral over $r$ in Eq. (A16) may be rewritten as follows:

$$
\begin{aligned}
\mathcal{Q}_{G}^{(l, l)}= & \left(i \eta_{1}-\frac{1}{2}\right) S^{(l)}+i p_{1} \int_{0}^{\infty} d r r^{3} I_{p_{2} l}(r) R_{p_{1} l}(r) \\
& -a_{1} A_{p_{1} l} \int_{0}^{\infty} d r r^{l+2} I_{p_{2} l}(r) e^{-i p_{1} r} \Phi\left(a_{2} ; c_{2} ; 2 i p_{1} r\right),
\end{aligned}
$$

where

$$
\begin{aligned}
S^{(l)}= & \int_{0}^{\infty} d r^{\prime} r^{\prime 2} u_{p_{2} l}\left(r^{\prime}\right) R_{1 s}\left(r^{\prime}\right) \\
& \times \int_{0}^{\infty} d r r^{2} R_{p_{1} l}(r) g_{l}\left(\mathcal{E}_{G} ; r, r^{\prime}\right) \\
= & \left(E_{p_{1}}-\mathcal{E}_{G}\right)^{-1} \int_{0}^{\infty} d r^{\prime} r^{\prime 2} R_{p_{1} l}(r) u_{p_{2} l}\left(r^{\prime}\right) R_{1 s}\left(r^{\prime}\right) .
\end{aligned}
$$

Equation (A24) is obtained by using the known relation $\left\langle R_{p l}(r)\right| g_{l}\left(\mathcal{E} ; r, r^{\prime}\right)=\left(E_{p}-\mathcal{E}\right)^{-1}\left\langle R_{p l}\left(r^{\prime}\right)\right|$. The expression for $S^{(l)}$ in Eq. (A24) was obtained in Appendix A 2 of Ref. [16].

The final result for $\mathcal{Q}_{G}^{(l, l)}\left(p_{1}, p_{2}\right)$ is

$$
\begin{aligned}
\mathcal{Q}_{G}^{(l, l)}= & \left(i \eta_{1}-\frac{1}{2}\right) S^{(l)}+2^{2 l+5} \nu(l+1) Z_{s}^{3} A_{p_{1} l} \mathcal{C}_{p_{2} l} \\
& \times \int_{0}^{1} d u u^{l-Z v} \int_{0}^{1} d x \frac{x^{l+i \eta_{2}}(1-x)^{l-i \eta_{2}}}{\tau^{2 l+3}} \\
& \times\left\{\frac{i p_{1}}{\alpha^{2}} K_{2 l, \beta_{1}}^{a_{1}, c_{2}, 2}\left(\lambda_{1}\right)-\frac{a_{1}}{\alpha^{2}} K_{2 l, \beta_{1}}^{a_{2}, c_{2}, 1}\left(\lambda_{1}\right)\right. \\
& \left.-\sum_{k=-1}^{2 l} b_{k}^{(l)}\left[i p_{1} K_{k, \beta_{2}}^{a_{1}, c_{2}, 2}\left(\lambda_{2}\right)-a_{1} K_{k, \beta_{2}}^{a_{2}, c_{2}, 1}\left(\lambda_{2}\right)\right]\right\},
\end{aligned}
$$

where $\lambda_{1,2}$ are defined in Eq. (A21). The results for $K_{k, \beta}^{\kappa, m, n}(\sigma)$ for $n=1,2$ are expressed in terms of $\mathcal{L}_{k, \beta}^{\kappa, m}(\sigma)$ using Eqs. (A14) and (A15),

$$
\begin{gathered}
K_{k, \beta}^{\kappa, m, 1}(\sigma)=-\left[v \mathcal{L}_{k, \beta}^{\kappa, m}(\sigma)+w \mathcal{L}_{k+1, \beta}^{\kappa+1, m+1}(\sigma)\right] \\
K_{k, \beta}^{\kappa, m, 2}(\sigma)=v^{\prime} \mathcal{L}_{k, \beta}^{\kappa, m}(\sigma)+w^{\prime} \mathcal{L}_{k+1, \beta}^{\kappa+1, m+1}(\sigma)-v K_{k, \beta}^{\kappa, m, 1}(\sigma) \\
-w K_{k+1, \beta}^{\kappa+1, m+1,1}(\sigma),
\end{gathered}
$$

where

$$
\begin{aligned}
& v=\frac{k-m+\kappa}{\sigma}-\frac{k \nu(1-u)}{\sigma \nu(1-\dot{u})+\beta}-\frac{\kappa}{\sigma-2 i p_{1}}, \\
& w=z^{\prime}[\sigma \nu(1-u)+\beta] \frac{\kappa k\left(\sigma-2 i p_{1}\right)}{m^{2} \sigma}, \\
& v^{\prime}=\frac{\kappa}{\left(\sigma-2 i p_{1}\right)^{2}}-\frac{k-m+\kappa}{\sigma^{2}}+\frac{k \nu^{2}(1-u)^{2}}{[\sigma \nu(1-u)+\beta]^{2}}, \\
& w^{\prime}=\frac{\kappa k\left(\sigma-2 i p_{1}\right)}{m^{2} \sigma}[[\sigma \nu(1-u)+\beta] \\
& \left.\times\left(z^{\prime \prime}+\frac{2 i p_{1} z^{\prime}}{\sigma\left(\sigma-2 i p_{1}\right)}\right)+z^{\prime} \nu(1-u)\right] \\
& z^{\prime}=2 i p_{1} \beta \frac{2 \nu(1-u)\left(\sigma-i p_{1}\right)+\beta}{[\sigma \nu(1-u)+\beta]^{2}\left(\sigma-2 i p_{1}\right)^{2}}, \\
& z^{\prime \prime}=-\frac{4 i p_{1} \beta}{\left(\sigma-2 i p_{1}\right)[\sigma \nu(1-u)+\beta]}\left[\frac{1}{\left(\sigma-2 i p_{1}\right)^{2}}\right. \\
& \left.+\frac{\nu(1-u)}{\left(\sigma-2 i p_{1}\right)[\sigma \nu(1-u)+\beta]}+\frac{\nu^{2}(1-u)^{2}}{[\sigma \nu(1-u)+\beta]^{2}}\right],
\end{aligned}
$$

and the primes in Eqs. (A26) and (A27) denote differentiation with respect to $\sigma$.

\section{c. Case $l_{1}=l, l_{2}=l+2$}

For this case, we obtain first the result

$$
\begin{aligned}
\hat{Q}^{\prime}(l, l+ & 2) R_{p_{1} l}(r) \\
= & i p_{1} A_{p_{1} l} r^{l} e^{-i p_{1} r} \\
& \times\left[\Phi\left(a_{1} ; c_{2} ; 2 i p_{1} r\right)+2 \frac{a_{1}}{c_{2}} \Phi\left(a_{2} ; c_{3} ; 2 i p_{1} r\right)\right] .
\end{aligned}
$$

Because the second parameter in the functions $\Phi$ in Eq. (A11) is equal to $c_{6}=2 l+6$, the value of the second parameter in the functions $\Phi$ in Eq. (A28) must be raised to become equal to $c_{6}$ by using the formulas

$$
\begin{aligned}
\Phi(c-3)= & \frac{1}{[c-3]_{2}}\left\{[a]_{2} \Phi(a+3)-3[a]_{1}(a-c+3)\right. \\
& \times \Phi(a+2)+3 a[a-c+2]_{1} \Phi(a+1) \\
& \left.-[a-c+1]_{2} \Phi\right\}, \\
\Phi(c-4)= & \frac{1}{[c-4]_{3}}\left\{[a]_{3} \Phi(a+4)-4[a]_{2}(a-c+4)\right. \\
& \times \Phi(a+3)+6[a]_{1}[a-c+3]_{1} \Phi(a+2) \\
& \left.-4 a[a-c+2]_{2} \Phi(a+1)+[a-c+1]_{3} \Phi\right\},
\end{aligned}
$$


which are obtained by repeated application of the relation [42]

$$
(c-1) \Phi(c-1)=a \Phi(a+1)-(a-c+1) \Phi .
$$

This yields

$$
\begin{aligned}
\hat{Q}^{\prime}(l, l+2) R_{p 1} l(r)= & i p 1 A_{p_{1}} l r^{l} e^{-i p_{1} r} \\
& \times \frac{1}{\left[c_{2}\right]_{3}}\left\{-\left[a_{1}\right]_{3} \Phi\left(a_{5} ; c_{6} ; 2 i p_{1} r\right)\right. \\
& +2\left[a_{1}\right]_{2}\left(a_{1}-c_{2}\right) \Phi\left(a_{4} ; c_{6} ; 2 i p_{1} r\right) \\
& -2 a_{1}\left[a_{1}-c_{4}\right]_{2} \Phi\left(a_{2} ; c_{6} ; 2 i p_{1} r\right) \\
& \left.+\left[a_{1}-c_{5}\right]_{3} \Phi\left(a_{1} ; c_{6} ; 2 i p_{1} r\right)\right\} .
\end{aligned}
$$

The final result for $\mathcal{Q}_{G}^{(l, l+2)}$ can then be written immediately by using Eq. (A13) for $n=0$,

$$
\begin{aligned}
\mathcal{Q}_{G}^{(l, l+2)}= & i \frac{2^{2 l+9}}{\left[c_{2}\right]_{3}} p_{1} \nu(l+3) Z_{s}^{3} A_{p_{1}} l \mathcal{C}_{p_{2} l+2} \\
& \times \int_{0}^{1} d u u^{l+2-Z v} \int_{0}^{1} d x \frac{x^{l+2+i \eta_{2}}(1-x)^{l+2-i \eta_{2}}}{\tau^{2 l+7}} \\
& \times\left\{\frac { 1 } { \alpha ^ { 2 } } \left[\delta_{5} \mathcal{L}_{c_{4}, \beta_{1}}^{a_{5}, c_{6}}\left(\lambda_{1}\right)+\delta_{4} \mathcal{L}_{c_{4}, \beta_{1}}^{a_{4}, c_{6}}\left(\lambda_{1}\right)+\delta_{2} \mathcal{L}_{c_{4}, \beta_{1}}^{a_{2}, c_{6}}\left(\lambda_{1}\right)\right.\right. \\
& \left.+\delta_{1} \mathcal{L}_{c_{4}, \beta_{1}}^{a_{1}, c_{6}}\left(\lambda_{1}\right)\right]-\sum_{k=-1}^{2 l+4} b_{k}^{(l+2)}\left[\delta_{5} \mathcal{L}_{k, \beta_{2}}^{a_{5}, c_{6}}\left(\lambda_{2}\right)\right. \\
& \left.\left.+\delta_{4} \mathcal{L}_{k, \beta_{2}}^{a_{4}, c_{6}}\left(\lambda_{2}\right)+\delta_{2} \mathcal{L}_{k, \beta_{2}}^{a_{2}, c_{6}}\left(\lambda_{2}\right)+\delta_{1} \mathcal{L}_{k, \beta_{2}}^{a_{1}, c_{6}}\left(\lambda_{2}\right)\right]\right\},
\end{aligned}
$$

where $\delta_{5}=-\left[a_{1}\right]_{3}, \delta_{4}=2\left[a_{1}\right]_{2}\left(a_{1}-c_{2}\right), \delta_{2}=-2 a_{1}\left[a_{1}-c_{4}\right]_{2}$, and $\delta_{1}=\left[a_{1}-c_{5}\right]_{3}$. To summarize, for all pairs of angular momenta, the evaluation of the quadrupole radial matrix elements reduces to the numerical calculation of twodimensional integrals of elementary functions.
[1] B. Krässig, M. Jung, D. S. Gemmell, E. P. Kanter, T. LeBrun, S. H. Southworth, and L. Young, Phys. Rev. Lett. 75, 4736 (1995).

[2] O. Hemmers, R. Guillemin, E. P. Kanter, B. Krässig, D. W. Lindle, S. H. Southworth, R. Wehlitz, J. Baker, A. Hudson, M. Lotrakul, D. Rolles, W. C. Stolte, I. C. Tran, A. Wolska, S. W. Yu, M. Ya. Amusia, K. T. Cheng, L. V. Chernysheva, W. R. Johnson, and S. T. Manson, Phys. Rev. Lett. 91, 053002 (2003).

[3] S. Ricz, R. Sankari, A. Köver, M. Jurvansuu, D. Varga, J. Nikkinen, T. Ricsoka, H. Aksela, and S. Aksela, Phys. Rev. A 67, 012712 (2003).

[4] O. Hemmers, R. Guillemin, D. Rolles, A. Wolska, D. W. Lindle, K. T. Cheng, W. R. Johnson, H. L. Zhou, and S. T. Manson, Phys. Rev. Lett. 93, 113001 (2004).

[5] E. P. Kanter, B. Krässig, S. H. Southworth, R. Guillemin, O. Hemmers, D. W. Lindle, R. Wehlitz, M. Ya. Amusia, L. V. Chernysheva, and N. L. S. Martin, Phys. Rev. A 68, 012714 (2003).

[6] A. Derevianko, O. Hemmers, S. Oblad, P. Glans, H. Wang, S. B. Whitfield, R. Wehlitz, I. A. Sellin, W. R. Johnson, and D. W. Lindle, Phys. Rev. Lett. 84, 2116 (2000).

[7] N. A. Cherepkov and S. K. Semenov, J. Phys. B 34, L211 (2001).

[8] T. Khalil, B. Schmidtke, M. Drescher, N. Müller, and U. Heinzmann, Phys. Rev. Lett. 89, 053001 (2002).

[9] A. N. Grum-Grzhimailo, J. Phys. B 34, L359 (2001).

[10] J. S. Briggs and V. Schmidt, J. Phys. B 33, R1 (2000).

[11] G. C. King and L. Avaldi, J. Phys. B 33, R215 (2000).

[12] A. Knapp, A. Kheifets, I. Bray, Th. Weber, A. L. Landers, S. Schössler, T. Jahnke, J. Nickles, S. Kammer, O. Jagutzki, L. Ph. H. Schmidt, T. Osipov, J. Rösch, M. H. Prior, H. SchmidtBöcking, C. L. Cocke, and R. Dörner, Phys. Rev. Lett. 89,
033004 (2002).

[13] S. Keller, J. Phys. B 33, L513 (2000).

[14] A. Y. Istomin, N. L. Manakov, and A. F. Starace, J. Phys. B 35, L543 (2002).

[15] T. Schneider and J. M. Rost, Phys. Rev. A 67, 062704 (2003).

[16] A. Y. Istomin, N. L. Manakov, and A. F. Starace, Phys. Rev. A 69, 032713 (2004).

[17] M. A. Kornberg and J. E. Miraglia, Phys. Rev. A 52, 2915 (1995).

[18] A. I. Mikhailov, I. A. Mikhailov, A. N. Moskalev, A. V. Nefiodov, G. Plunien, and G. Soff, Phys. Lett. A 316, 395 (2003).

[19] A. I. Mikhailov, I. A. Mikhailov, A. N. Moskalev, A. V. Nefiodov, G. Plunien, and G. Soff, Phys. Rev. A 69, 032703 (2004).

[20] J. Berakdar and H. Klar, Phys. Rev. Lett. 69, 1175 (1992).

[21] J. Viefhaus, L. Avaldi, G. Snell, M. Wiedenhöft, R. Hentges, A. Rüdel, F. Schäfers, D. Menke, U. Heinzmann, A. Engelns, J. Berakdar, H. Klar, and U. Becker, Phys. Rev. Lett. 773975 (1996).

[22] J. Berakdar and H. Klar, Phys. Rep. 340, 474 (2001).

[23] J. Berakdar, H. Klar, A. Huetz, and P. Selles, J. Phys. B 26, 1463 (1993).

[24] N. L. Manakov, S. I. Marmo, and A. V. Meremianin, J. Phys. B 29, 2711 (1996).

[25] L. Malegat, P. Selles, and A. Huetz, J. Phys. B 30, 251 (1997).

[26] A. S. Kheifets and I. Bray, Phys. Rev. Lett. 81, 4588 (1998).

[27] J. Berakdar, J. Phys. B 31, 3167 (1998); 32, L27 (1999).

[28] A. S. Kheifets, I. Bray, K. Soejima, A. Danjo, K. Okuno, and A. Yagishita, J. Phys. B 32, L501 (1999).

[29] S. A. Collins, S. Cvejanović, C. Dawson, T. J. Reddish, D. P. Seccombe, A. Huetz, L. Malegat, P. Selles, A. K. Kazansky, A. Danjo, K. Soejima, K. Okuno, and A. Yagishita, Phys. Rev. A 65, 052717 (2002). 
[30] V. Mergel, M. Achler, R. Dörner, K. Khayyat, T. Kambara, Y. Awaya, V. Zoran, B. Nyström, L. Spielberger, J. H. McGuire, J. Feagin, J. Berakdar, Y. Azuma, and H. Schmidt-Böcking, Phys. Rev. Lett. 80, 5301 (1998).

[31] K. Soejima, A. Danjo, K. Okuno, and A. Yagishita, Phys. Rev. Lett. 83, 1546 (1999).

[32] M. Achler, V. Mergel, L. Spielberg, R. Dörner, Y. Azuma, and H. Schmidt-Böcking, J. Phys. B 34, 965 (2001).

[33] A. Y. Istomin, N. L. Manakov, A. V. Meremianin, and A. F. Starace, Phys. Rev. Lett. 92, 063002 (2004).

[34] A. Y. Istomin, N. L. Manakov, A. V. Meremianin, and A. F. Starace, Phys. Rev. A 70, 010702(R) (2004).

[35] A. I. Akhiezer and V. B. Berestetskii, Quantum Electrodynamics (Wiley, New York, 1974).

[36] D. A. Varshalovich, A. N. Moskalev, and V. K. Khersonskii,
Quantum Theory of Angular Momentum (World Scientific, Singapore, 1988).

[37] P. Lablanquie, J. Mazeau, L. Andric, P. Selles, and A. Huetz, Phys. Rev. Lett. 74, 2192 (1995).

[38] L. Hostler, J. Math. Phys. 5, 591 (1964).

[39] H. Bräuning, R. Dörner, C. L. Cocke, M. H. Prior, B. Krässig, A. S. Kheifets, I. Bray, A. Bräuning-Demian, K. Carnes, S. Dreuil, V. Mergel, P. Richard, J. Ullrich, and H. SchmidtBöcking, J. Phys. B 31, 5149 (1998).

[40] A. Knapp (private communication).

[41] J. Colgan and M. S. Pindzola, J. Phys. B 37, 1153 (2004).

[42] A. Erdelyi, W. Magnus, F. Oberhettinger, and F. G. Tricomi, Higher Transcendental Functions (McGraw-Hill, New York, 1953), Vol. 1. 